JOURNAL OF THE

AMERICAN MATHEMATICAL SOCIETY

Volume 25, Number 3, July 2012, Pages 739-757

S 0894-0347(2012)00728-0

Article electronically published on January 10, 2012

\title{
A GENERALIZATION OF STEINBERG'S CROSS SECTION
}

\author{
XUHUA HE AND GEORGE LUSZTIG
}

\section{INTRODUCTION}

0.1. Let $G$ be a connected semisimple algebraic group over an algebraically closed field. Let $B, B^{-}$be two opposed Borel subgroups of $G$ with unipotent radicals $U, U^{-}$and let $T=B \cap B^{-}$, a maximal torus of $G$. Let $N T$ be the normalizer of $T$ in $G$ and let $W=N T / T$ be the Weyl group of $T$, a finite Coxeter group with length function $l$. For $w \in W$ let $\dot{w}$ be a representative of $w$ in $N T$. The following result is due to Steinberg [St, 8.9] (but the proof in loc.cit. is omitted): if $w$ is a Coxeter element of minimal length in $W$, then (i) the conjugation action of $U$ on $U \dot{w} U$ has trivial isotropy groups and (ii) the subset $\left(U \cap \dot{w} U^{-} \dot{w}^{-1}\right) \dot{w}$ meets any $U$-orbit on $U \dot{w} U$ in exactly one point; in particular, (iii) the set of $U$-orbits on $U \dot{w} U$ is naturally an affine space of dimension $l(w)$.

More generally, assuming that $w$ is any elliptic element of $W$ of minimal length in its conjugacy class, it is shown in [L3] that (i) holds and, assuming in addition that $G$ is of classical type, it is shown in [L5] that (iii) holds. In this paper we show for any $w$ as above and any $G$ that (ii) (and hence (iii)) hold; see 3.6(ii) (actually we take $\dot{w}$ of a special form but then the result holds in general since any representative of $w$ in $N T$ is of the form $t \dot{w} t^{-1}$ for some $t \in T$ ). We also prove analogous statements in some twisted cases, involving an automorphism of the root system or a Frobenius map (see Theorem 3.6) and a version over $\mathbf{Z}$ of these statements using the results in [L2] on groups over $\mathbf{Z}$. Note that the proof of (ii) given in this paper uses (as does the proof of (i) in [L3]) a result in [GP, 3.2.7] and a weak form of the existence of "good elements" GM in an elliptic conjugacy class in $W$.

0.2. Let $w$ be an elliptic element of $W$ which has minimal length in its conjugacy class $C$ and let $\gamma$ be the unipotent class of $G$ attached to $C$ in L3. Recall that $\gamma$ has codimension $l(w)$ in $G$. As an application of our results we construct (see 4.2(a)) a closed subvariety $\Sigma$ of $G$ isomorphic to an affine space of dimension $l(w)$ such that $\Sigma \cap \gamma$ is a finite set with a transitive action of a certain finite group whose order is divisible only by the bad primes of $G$. In the case where $C$ is the Coxeter class, $\Sigma$ reduces to Steinberg's cross section $[\mathrm{St}]$ which intersects the regular unipotent class in $G$ in exactly one element.

Received by the editors March 14, 2011 and, in revised form, October 4, 2011, and December $5,2011$.

2010 Mathematics Subject Classification. Primary 20G99.

The first author was supported in part by HKRGC grant 601409 .

The second author was supported in part by National Science Foundation grant DMS-0758262.

(C)2012 American Mathematical Society Reverts to public domain 28 years from publication 
0.3. Recently, A. Sevostyanov $\mathrm{Se}$ proved statements similar to (i),(ii),(iii) in $\S 0.1$ for a certain type of Weyl group elements assuming that the ground field is $\mathbf{C}$. It is not clear to us what is the relation of the Weyl group elements considered in $\mathrm{Se}$ with those considered in this paper.

0.4. The following (unpublished) example of N. Spaltenstein, dating from the late 1970s, shows that the statement (i) (for Coxeter elements) in $\S 0.1$ can be false if the assumption of minimal length is dropped: the elements

$$
\begin{aligned}
& \dot{w}=\left(\begin{array}{cccccc}
0 & 0 & 1 & 0 & 0 & 0 \\
0 & 0 & 0 & 0 & 1 & 0 \\
0 & 1 & 0 & 0 & 0 & 0 \\
0 & 0 & 0 & 0 & 0 & 1 \\
0 & 0 & 0 & 1 & 0 & 0 \\
1 & 0 & 0 & 0 & 0 & 0
\end{array}\right), \quad u_{x}=\left(\begin{array}{cccccc}
1 & 0 & 0 & 0 & 0 & 0 \\
0 & 1 & 0 & x & 0 & x \\
0 & 0 & 1 & x & 0 & x \\
0 & 0 & 0 & 1 & 0 & 0 \\
0 & 0 & 0 & 0 & 1 & 0 \\
0 & 0 & 0 & 0 & 0 & 1
\end{array}\right), \\
& y=\left(\begin{array}{cccccc}
1 & 0 & -1 & 0 & 0 & 0 \\
0 & 1 & 1 & 0 & 0 & 0 \\
0 & 0 & 1 & 0 & 0 & 0 \\
0 & 0 & 0 & 1 & 1 & -1 \\
0 & 0 & 0 & 0 & 1 & 0 \\
0 & 0 & 0 & 0 & 0 & 1
\end{array}\right)
\end{aligned}
$$

of $G L_{6}(\mathbf{C})$ (with $x \in \mathbf{C}$ ) satisfy $u_{x} y \dot{w} u_{x}^{-1}=y \dot{w}$; hence if $U$ is the group of upper triangular matrices in $G L_{6}(\mathbf{C})$, then in the conjugation action of $U$ on $U \dot{w} U$, the isotropy group of $y \dot{w}$ contains the one-parameter group $\left\{u_{x} ; x \in \mathbf{C}\right\}$.

[Note added 10.4.2011. We thank Ulrich Goertz for pointing out that under the assumption of our Proposition 1.2, $f_{\mathbf{Z}}$ is an isomorphism of schemes (this strengthening of Proposition 1.2 will not be used here).]

\section{Polynomial maps of an affine space to itself}

1.1. Let $\mathcal{C}$ be the class of commutative rings with 1 . Let $N$ be an integer $\geq 1$. A family $\left(f_{A}\right)_{A \in \mathcal{C}}$ of maps $f_{A}: A^{N} \rightarrow A^{N}$ is said to be polynomial if there exist (necessarily unique) polynomials with integer coefficients

$$
f_{1}\left(X_{1}, \ldots, X_{N}\right), \ldots, f_{N}\left(X_{1}, \ldots, X_{N}\right)
$$

in the indeterminates $X_{1}, \ldots, X_{N}$ such that for any $A \in \mathcal{C}, f_{A}$ is the map

$$
\left(a_{1}, \ldots, a_{N}\right) \mapsto\left(f_{1}\left(a_{1}, \ldots, a_{N}\right), \ldots, f_{N}\left(a_{1}, \ldots, a_{N}\right)\right) .
$$

For such a family we define for any $A \in \mathcal{C}$ an $A$-algebra homomorphism $f_{A}^{*}$ : $A\left[X_{1}, \ldots, X_{N}\right] \rightarrow A\left[X_{1}, \ldots, X_{N}\right]$ by $f_{A}^{*}\left(X_{i}\right)=f_{i}\left(X_{1}, \ldots, X_{N}\right)$ for all $i \in[1, N]$ (here we view $f_{i}$ as an element of $A\left[X_{1}, \ldots, X_{N}\right]$ using the obvious ring homomorphism $\mathbf{Z} \rightarrow A$ ). We have the following result.

Proposition 1.2. Assume that $f_{A}: A^{N} \rightarrow A^{N}(A \in \mathcal{C})$ is a polynomial family such that $f_{A}$ is injective for any $A \in \mathcal{C}$. Then:

(i) $f_{A}$ is bijective in the following cases: (a) $A$ is finite; (b) $A=\mathbf{Z}_{p}$, the ring of $p$-adic integers ( $p$ is a prime number); (c) $A$ is a perfect field; (d) $A$ is the ring of rational numbers which have no $p$ in the denominator ( $p$ is a prime number); (e) $A=\mathbf{Z}$.

(ii) If $A$ is an algebraically closed field, then $f_{A}$ is an isomorphism of algebraic varieties. 
We prove (i). In case (a), $A^{N}$ is a finite set and the result follows.

Assume that $A$ is as in (b). For any $s \geq 1$ let $A_{s}=\mathbf{Z} / p^{s} \mathbf{Z}$ (a finite ring) and let $l_{s}: A \rightarrow A_{s}$ be the obvious homomorphism. Let $\xi \in A^{N}$. Let $\xi_{s}=l_{s}^{N}(\xi) \in A_{s}^{N}$. Using (a) for $A_{s}$ we set $\xi_{s}^{\prime}=f_{A_{s}}^{-1}\left(\xi_{s}\right) \in A_{s}^{N}$. Let $\xi^{\prime} \in A^{N}$ be the unique element such that for any $s \geq 1$, the image of $\xi^{\prime}$ under $l_{s}^{N}: A^{N} \rightarrow A_{s}^{N}$ is equal to $\xi_{s}^{\prime}$. Let $\tilde{\xi}=f_{A}\left(\xi^{\prime}\right)$. Then for any $s \geq 1, \xi, \tilde{\xi}$ have the same image under $l_{s}^{N}: A^{N} \rightarrow A_{s}^{N}$. Hence $\tilde{\xi}=\xi$. Thus $f_{A}$ is surjective, as desired.

Assume that $A$ is as in (c). Let $A^{\prime}$ be an algebraic closure of $A$. By BR (see also $[\mathrm{Ax}, \mathrm{G1}, 10.4 .11]), f_{A^{\prime}}$ is bijective. Let $\xi \in A^{N}$. Since $A^{N} \subset A^{\prime N}$, we can view $\xi$ as an element of $A^{\prime N}$ so that $\xi^{\prime}=f_{A^{\prime}}^{-1}(\xi) \in A^{\prime N}$ is defined. For any $\gamma \in \operatorname{Gal}\left(A^{\prime} / A\right)$ we have

$$
f_{A^{\prime}}\left(\gamma\left(\xi^{\prime}\right)\right)=\gamma\left(f_{A^{\prime}}\left(\xi^{\prime}\right)\right)=\gamma(\xi)=\xi=f_{A^{\prime}}\left(\xi^{\prime}\right) .
$$

(The obvious action of $\gamma$ on $A^{\prime N}$ is denoted again by $\gamma$.) Using the injectivity of $f_{A^{\prime}}$ we deduce that $\gamma\left(\xi^{\prime}\right)=\xi^{\prime}$. Since this holds for any $\gamma$ and $A$ is perfect, it follows that $\xi^{\prime} \in A^{N}$. We have $f_{A}\left(\xi^{\prime}\right)=\xi$. Thus $f_{A}$ is surjective, as desired.

Assume that $A$ is as in (d). Let $A_{0}=\mathbf{Q}_{p}$, the field of $p$-adic numbers. We can view $A$ as the intersection of two subrings of $A_{0}$, namely $A_{1}=\mathbf{Q}$ and $A_{2}=\mathbf{Z}_{p}$. Now $f_{A_{i}}$ is bijective for $i=0,1,2$ by (b),(c). Let $\xi \in A^{N}$. For $i=0,1,2$ we set $\xi_{i}^{\prime}=f_{A_{i}}^{-1}(\xi) \in A_{i}^{N}$. Clearly, $\xi_{1}^{\prime}=\xi_{0}^{\prime}=\xi_{2}^{\prime}$; hence $\xi_{0}^{\prime} \in A^{N}$ and $f_{A}\left(\xi_{0}^{\prime}\right)=\xi$. Thus $f_{A}$ is surjective, as desired.

Assume that $A=\mathbf{Z}$. We can view $A$ as $\bigcap_{p} A_{p}$, where $p$ runs over the set of prime numbers and $A_{p}$ is the ring denoted by $A$ in (d); the intersection is taken in the field Q. Now $f_{A_{p}}$ is bijective for any $p$ (see (d)) and $f_{\mathbf{Q}}$ is bijective by (c). Let $\xi \in A^{N}$. We set $\xi_{p}^{\prime}=f_{A_{p}}^{-1}(\xi) \in A_{p}^{N}$ and $\xi^{\prime}=f_{\mathbf{Q}}^{-1}(\xi) \in \mathbf{Q}^{N}$. Clearly, $\xi_{p}^{\prime}=\xi^{\prime}$ for all $p$. Hence $\xi^{\prime} \in A^{N}$ and $f_{A}\left(\xi^{\prime}\right)=\xi$. Thus $f_{A}$ is surjective, as desired. This proves (i).

Now assume that $A$ is as in (ii). Since $f_{A_{1}}: A_{1}^{N} \rightarrow A_{1}^{N}$ is bijective for any algebraically closed field $A_{1}$ (see (i)), it is enough to show that the morphism $f_{A}$ is étale (see [G2, 17.9.1]). Let $A^{\prime}=A \oplus A$, regarded as an $A$-algebra with multiplication $(a, b)\left(a^{\prime}, b\right)=\left(a b, a b^{\prime}+a^{\prime} b\right)$. The unit element is $1=(1,0)$. We set $T=(0,1)$. Then $(a, b)=a+b T$ and $T^{2}=0$. Then $f_{A^{\prime}}$ is defined. There exist polynomials $f_{k}\left(X_{1}, \ldots, X_{N}\right)(k \in[1, N])$ with coefficients in $\mathbf{Z}$ such that

$$
\begin{aligned}
& f_{A^{\prime}}\left(a_{1}+b_{1} T, \ldots, a_{N}+b_{N} T\right) \\
& =\left(f_{1}\left(a_{1}+b_{1} T, \ldots, a_{N}+b_{N} T\right), \ldots, f_{N}\left(a_{1}+T, \ldots, a_{N}+T b_{N}\right)\right) \\
& =\left(f_{1}\left(a_{*}\right)+\sum_{k=1}^{N} \frac{\partial f_{1}}{\partial X_{k}}\left(a_{*}\right) b_{k} T, \ldots, f_{N}\left(a_{*}\right)+\sum_{k=1}^{N} \frac{\partial f_{N}}{\partial X_{k}}\left(a_{*}\right) b_{k} T\right)
\end{aligned}
$$

for any $a_{*}=\left(a_{1}, \ldots, a_{N}\right) \in A^{N}, b_{*}=\left(b_{1}, \ldots, b_{N}\right) \in A^{N}$. Since $f_{A^{\prime}}$ is injective, we see that for any $a_{*} \in A^{N}$, the (linear) map $A^{N} \rightarrow A^{N}$ given by

$$
b_{*} \mapsto\left(\sum_{k=1}^{N} \frac{\partial f_{1}}{\partial X_{k}}\left(a_{*}\right) b_{k}, \ldots, \sum_{k=1}^{N} \frac{\partial f_{N}}{\partial X_{k}}\left(a_{*}\right) b_{k}\right)
$$

is injective. It follows that for any $a_{*} \in A^{N}$, the $N \times N$ matrix with $(j, k)$-entries $\frac{\partial f_{j}}{\partial X_{k}}\left(a_{*}\right)$ is nonsingular. This shows that $f_{A}$ is étale. This proves (ii). The proposition is proved. 
Proposition 1.3. Assume that $f_{A}: A^{N} \rightarrow A^{N}(A \in \mathcal{C})$ and $f_{A}^{\prime}: A^{N} \rightarrow A^{N}$ $(A \in \mathcal{C})$ are two polynomial families such that $f_{A}^{\prime} f_{A}=1$ for all $A \in \mathcal{C}$. Then for any $A \in \mathcal{C}, f_{A}^{*}$ is an A-algebra isomorphism and $f_{A}: A^{N} \rightarrow A^{N}$ is bijective.

Since $f_{A}^{\prime} f_{A}=1$ we have $f_{A}^{*} f_{A}^{\prime *}=1$ and $f_{A}$ is injective for any $A$. Using Proposition 1.2 we see that $f_{\mathbf{Z}}$ is bijective; hence $f_{\mathbf{Z}} f_{\mathbf{Z}}^{\prime}=1$. Let $\xi_{A}=f_{A} f_{A}^{\prime}$. Then $\left(\xi_{A}\right)_{A \in \mathcal{C}}$ is a polynomial family and $\xi_{\mathbf{Z}}=1$. Thus $\xi_{\mathbf{Z}}^{*}\left(X_{i}\right)=X_{i}$ for any $i$ (there is at most one element of $\mathbf{Z}\left[X_{1}, \ldots, X_{N}\right]$ with prescribed values at any $\left.\left(x_{1}, \ldots, x_{N}\right) \in \mathbf{Z}^{N}\right)$. We see that the polynomials with integer coefficients which define $\xi$ are $X_{1}, \ldots, X_{N}$. It follows that $\xi_{A}=1$ for any $A \in \mathcal{C}$; hence $f_{A} f_{A}^{\prime}=1$ and $f_{A}$ is a bijection. Also, since $\xi_{A}^{*}=1$ we have $f_{A}^{\prime *} f_{A}^{*}=1$ for any $A$; hence $f_{A}^{*}$ is an isomorphism. The proposition is proved.

\section{Reductive Groups Over A RING}

2.1. We fix a root datum $\mathcal{R}$ as in [L1, 2.2]. This consists of two free abelian groups of finite type $Y, X$ with a given perfect pairing $\langle\rangle:, Y \times X \rightarrow \mathbf{Z}$ and a finite set $I$ with given imbeddings $I \rightarrow Y \quad(i \mapsto i)$ and $I \rightarrow X \quad\left(i \mapsto i^{\prime}\right)$ such that $\left\langle i, i^{\prime}\right\rangle=2$ for all $i \in I$ and $\left\langle i, j^{\prime}\right\rangle \in-\mathbf{N}$ for all $i \neq j$ in $I$; in addition, we are given a symmetric bilinear form $\mathbf{Z}[I] \times \mathbf{Z}[I] \rightarrow \mathbf{Z}, \nu, \nu^{\prime} \mapsto \nu \cdot \nu^{\prime}$ such that $i \cdot i \in 2 \mathbf{Z}_{>0}$ for all $i \in I$ and $\left\langle i, j^{\prime}\right\rangle=2 i \cdot j / i \cdot i$ for all $i \neq j$ in $I$. We assume that the matrix $M=(i \cdot j)_{i, j \in I}$ is positive definite.

Let $W$ be the (finite) subgroup of $\operatorname{Aut}(X)$ generated by the involutions $s_{i}$ : $x-\langle i, x\rangle i^{\prime} \quad(i \in I)$. For $i \neq j$ in $I$, let $n_{i, j}=n_{j, i}$ be the order of $s_{i} s_{j}$ in $W$. Note that $W$ is a (finite) Coxeter group with generators $S:=\left\{s_{i} ; i \in I\right\}$; let $l: W \rightarrow \mathbf{N}$ be the standard length function. Let $w_{I}$ be the unique element of maximal length of $W$. For $J \subset I$ let $W_{J}$ be the subgroup of $W$ generated by $\left\{s_{i} ; i \in J\right\}$. Let $\mathcal{X}$ be the set of all sequences $i_{1}, i_{2}, \ldots, i_{n}$ in $I$ such that $s_{i_{1}} s_{i_{2}} \ldots s_{i_{n}}=w_{I}$ and $l\left(w_{I}\right)=n$.

2.2. Now (until the end of $\S 2.10$ ) we fix $A \in \mathcal{C}$. Let $\dot{\mathbf{U}}_{A}$ be the $A$-algebra attached to $\mathcal{R}$ and to $A$ (with $v=1$ ) in [L1, 31.1.1], where it is denoted by ${ }_{A} \dot{\mathbf{U}}$. As in loc.cit. we denote the canonical basis of the $A$-module $\dot{\mathbf{U}}_{A}$ by $\dot{\mathbf{B}}$. For $a, b, c \in \dot{\mathbf{B}}$ we define $m_{a, b}^{c} \in A, \hat{m}_{c}^{a, b} \in A$ as in [L2, 1.5]; in particular, we have $a b=\sum_{c \in \dot{\mathbf{B}}} m_{a, b}^{c} c$. Let $\hat{\mathbf{U}}_{A}$ be the $A$-module consisting of all formal linear combinations $\sum_{a \in \dot{\mathbf{B}}} n_{a} a$ with $n_{a} \in A$. There is a well-defined $A$-algebra structure on $\hat{\mathbf{U}}_{A}$ such that

$$
\left(\sum_{a \in \dot{\mathbf{B}}} n_{a} a\right)\left(\sum_{b \in \dot{\mathbf{B}}} \tilde{n}_{b} b\right)=\sum_{c \in \dot{\mathbf{B}}} r_{c} c
$$

where $r_{c}=\sum_{(a, b) \in \dot{\mathbf{B}} \times \dot{\mathbf{B}}} m_{a, b}^{c} n_{a} \tilde{n}_{b}$. (See [L2, 1.11].) It has unit element $1=$ $\sum_{\zeta \in X} 1_{\zeta}$, where for any $\zeta \in X$ the element $1_{\zeta} \in \dot{\mathbf{B}}$ is defined as in [L1, 31.1.1]. Let $\epsilon: \hat{\mathbf{U}}_{A} \rightarrow A$ be the algebra homomorphism given by $\sum_{a \in \dot{\mathbf{B}}} n_{a} a \mapsto n_{1_{0}}$. We identify $\dot{\mathbf{U}}_{A}$ with the subalgebra of $\hat{\mathbf{U}}_{A}$ consisting of finite $A$-linear combinations of elements in $\dot{\mathbf{B}}$.

Let $\mathbf{f}_{A}$ be the $A$-algebra with 1 associated to $M$ and $A$ (with $v=1$ ) in L1, 31.1.1], where it is denoted by ${ }_{A} \mathbf{f}$. Let $\mathbf{B}$ be the canonical basis of the $A$-module $\mathbf{f}_{A}$ (see [L1, 31.1.1]). For $i \in I, c \in \mathbf{N}$, the element $\theta_{i}^{(c)}$ (see [L1, 1.4.1, 31.1.1]) of $\mathbf{f}_{A}$ is contained in $\mathbf{B}$. Let $\left(x \otimes x^{\prime}\right): u \mapsto x^{-} u x^{\prime+}$ be the $\mathbf{f}_{A} \otimes_{A} \mathbf{f}_{A}^{o p p}$-module structure on $\dot{\mathbf{U}}_{A}$ considered in [L1, 31.1.2]; we write $u x^{\prime+}$ instead of $1^{-} u x^{\prime+}$ and $x^{-} u$ instead of $x^{-} u 1^{+}$. 
The elements $\left\{1_{\zeta} b^{+} ; b \in \mathbf{B}, \zeta \in X\right\}$ (resp. $\left\{b^{-} 1_{\zeta} ; b \in \mathbf{B}, \zeta \in X\right\}$ ) of $\dot{\mathbf{U}}_{A}$ are distinct and form a subset of $\dot{\mathbf{B}}$. Let $\hat{\mathbf{U}}_{A}^{+}\left(\right.$resp. $\left.\hat{\mathbf{U}}_{A}^{-}\right)$be the $A$-submodule of $\hat{\mathbf{U}}_{A}$ consisting of elements

$$
\sum_{b \in \mathbf{B}, \zeta \in X} n_{b}\left(1_{\zeta} b^{+}\right) \quad\left(\text { resp. } \sum_{b \in \mathbf{B}, \zeta \in X} n_{b}\left(b^{-} 1_{\zeta}\right)\right)
$$

with $n_{b} \in A$.

2.3. As in [L2, 4.1], let $G_{A}$ be the set of all $\sum_{a \in \dot{\mathbf{B}}} n_{a} a \in \hat{\mathbf{U}}_{A}$ such that $n_{1_{0}}=1$ and $\sum_{c \in \dot{\mathbf{B}}} \hat{m}_{c}^{a, b} n_{c}=n_{a} n_{b}$ for all $a, b \in \dot{\mathbf{B}}$ (the last sum is finite by [L2, 1.16]). Note that $G_{A}$ is a subgroup of the group of invertible elements of the algebra $\hat{\mathbf{U}}_{A}$. As in [L2, 4.2] we set $U_{A}=G_{A} \cap \hat{\mathbf{U}}_{A}^{+}, U_{A}^{-}=G_{A} \cap \hat{\mathbf{U}}_{A}^{-}$(in loc.cit. $U_{A}, U_{A}^{-}$are denoted by $\left.G_{A}^{>0}, G_{A}^{<0}\right)$. Let $T_{A}$ be the set of elements of $G_{A}$ of the form $\sum_{\lambda \in X} n_{\lambda} 1_{\lambda}$ with $n_{\lambda} \in A$. As shown in loc.cit., $U_{A}, U_{A}^{-}, T_{A}$ are subgroups of $G_{A}$. We note that

(a) multiplication in $G_{A}$ defines an injective map $U_{A}^{-} \times T_{A} \times U_{A} \rightarrow G_{A}$. This statement appears in L2, 4.2(a)] but the line

" $\hat{\mathbf{U}}_{A}^{-} \cap \hat{\mathbf{U}}_{A}^{>0}=\{1\}$. Thus, $\xi_{3}^{\prime} \xi_{3}^{-1}=1$ so that $\xi_{3}^{\prime}=\xi_{3}$." in the proof in loc. cit. should be replaced by:

" $\hat{\mathbf{U}}_{A}^{-} \cap \hat{\mathbf{U}}_{A}^{>0}=\{a 1 ; a \in A\}$. Thus, $\xi_{3}^{\prime} \xi_{3}^{-1}=a 1$ for some $a \in A$. Since $\epsilon\left(\xi_{3}^{\prime} \xi_{3}^{-1}\right)=1$, we have $a=1$ so that $\xi_{3}^{\prime}=\xi_{3}$."

From (a) we deduce that

(b) $U_{A} \cap U_{A}^{-}=\{1\}$.

2.4. For any $i \in I, h \in A$ we set

$$
\begin{aligned}
& x_{i}(h)=\sum_{c \in \mathbf{N}, \lambda \in X} h^{c} 1_{\lambda} \theta_{i}^{(c)+} \in \hat{\mathbf{U}}_{A}, \\
& y_{i}(h)=\sum_{c \in \mathbf{N}, \lambda \in X} h^{c} \theta_{i}^{(c)-} 1_{\lambda} \in \hat{\mathbf{U}}_{A} .
\end{aligned}
$$

By [L2, 1.18(a)] we have $x_{i}(h) \in U_{A}, y_{i}(h) \in U_{A}^{-}$. For any $i \in I$ we set

$$
\dot{s}_{i}=\sum_{a \in \mathbf{N}, b \in \mathbf{N}, \lambda \in X ;\langle i, \lambda\rangle=a+b}(-1)^{a} \theta_{i}^{(a)-} 1_{\lambda} \theta_{i}^{(b)+} \in \hat{\mathbf{U}}_{A} .
$$

(Note that $\theta_{i}^{(a)-} 1_{\lambda} \theta_{i}^{(b)+} \in \dot{\mathbf{B}}$ whenever $a+b=\langle i, \lambda\rangle$.) By [L2, 2.2(e)] we have $\dot{s}_{i} \in G_{A}$. (In loc.cit., $\dot{s}_{i}$ is denoted by $s_{i, 1}^{\prime \prime}$.) From [L2, 2.4] we see that if $i, j \in I$, $i \neq j$, then

(a) $\dot{s}_{i} \dot{s}_{j} \dot{s}_{i} \ldots=\dot{s}_{j} \dot{s}_{i} \dot{s}_{j} \ldots$ in $G_{A}$ (both products have $n_{i, j}$ factors).

For $i \in I$ we have

(b) $\dot{s}_{i}^{2}=t_{i}(-1)$, where $t_{i}(-1)=\sum_{\lambda \in X}(-1)^{\langle i, \lambda\rangle} 1_{\lambda} \in T_{A}$ normalizes $U_{A}, U_{A}^{-}$. (See [L2, 2.3(b), 4.4(a), 4.3(a)].)

For $i \in I, h \in A$ we have

(c) $\dot{s}_{i}^{-1} x_{i}(h) \dot{s}_{i}=y_{i}(-h)$.

This follows from the definitions using [L2, 2.3(c)].

For any $w \in W$ we set $\dot{w}=\dot{s}_{i_{1}} \dot{s}_{i_{2}} \ldots \dot{s}_{i_{k}} \in G_{A}$, where $i_{1}, \ldots, i_{k}$ in $I$ are such that $s_{i_{1}} s_{i_{2}} \ldots s_{i_{k}}=w, k=l(w)$. This is well defined, by (a). 
2.5. We have the following result (see [L2, 4.7(a)]):

(a) Let $w \in W$ and let $i \in I$ be such that $l\left(w s_{i}\right)=l(w)+1$. Let $h \in A$. We have $\dot{w} x_{i}(h) \dot{w}^{-1} \in U_{A}$.

The proof of the following result is similar to that of (a):

(b) Let $z \in W$ and let $i \in I$ be such that $l\left(s_{i} z\right)=l(z)+1$. Let $h \in A$. Then we have $\dot{z}^{-1} y_{i}(h) \dot{z} \in U_{A}^{-}$.

2.6. Let $\left(i_{1}, \ldots, i_{n}\right) \in \mathcal{X}$. For any $\left(h_{1}, h_{2}, \ldots, h_{n}\right) \in A^{n}$ we have

$$
\begin{aligned}
& x_{i_{1}}\left(h_{1}\right) \dot{s}_{i_{1}} x_{i_{2}}\left(h_{2}\right) \dot{s}_{i_{2}} \ldots x_{i_{n}}\left(h_{n}\right) \dot{s}_{i_{n}} \dot{w}_{I}^{-1} \\
& =x_{i_{1}}\left(h_{1}\right)\left(\dot{s}_{i_{1}} x_{i_{2}}\left(h_{2}\right) \dot{s}_{i_{1}}^{-1}\right) \ldots\left(\dot{s}_{i_{1}} \dot{s}_{i_{2}} \ldots \dot{s}_{i_{n-1}} x_{i_{n}}\left(h_{n}\right) \dot{s}_{i_{n-1}}^{-1} \ldots \dot{s}_{i_{2}}^{-1} \dot{s}_{i_{1}}^{-1}\right) \in U_{A} .
\end{aligned}
$$

(We use 2.5(a).) From [L2, 4.8(a)] we see that

(a) the map $A^{n} \rightarrow U_{A},\left(h_{1}, h_{2}, \ldots, h_{n}\right) \mapsto x_{i_{1}}\left(h_{1}\right) \dot{s}_{i_{1}} x_{i_{2}}\left(h_{2}\right) \dot{s}_{i_{2}} \ldots x_{i_{n}}\left(h_{n}\right) \dot{s}_{i_{n}} \dot{w}_{I}^{-1}$ is a bijection.

2.7. Let $w \in W$. Let

$$
U_{A}^{w}=U_{A} \cap \dot{w} U_{A}^{-} \dot{w}^{-1}, \quad{ }^{w} U_{A}=U_{A} \cap \dot{w} U_{A} \dot{w}^{-1} ;
$$

these are subgroups of $U_{A}$. We can find $\left(i_{1}, \ldots, i_{n}\right) \in \mathcal{X}$ such that $s_{i_{1}} s_{i_{2}} \ldots s_{i_{k}}=w$, where $k=l(w)$. Let $\left(h_{1}, h_{2}, \ldots, h_{n}\right) \in A^{n}$ and let $u \in U_{A}$ be its image under the map 2.6(a). We have $u=u^{\prime} u^{\prime \prime}$, where $u^{\prime}=r_{1} r_{2} \ldots r_{k}, u^{\prime \prime}=r_{k+1} r_{k+2} \ldots r_{n}$ and

$$
r_{m}=\dot{s}_{i_{1}} \dot{s}_{i_{2}} \ldots \dot{s}_{i_{m-1}} x_{i_{m}}\left(h_{m}\right) \dot{s}_{i_{m-1}}^{-1} \ldots \dot{s}_{i_{2}}^{-1} \dot{s}_{i_{1}}^{-1} \in U_{A}
$$

for $m \in[1, n]$. If $m \in[1, k]$ we have

$$
\begin{aligned}
& \dot{w}^{-1} r_{m} \dot{w}=\dot{s}_{i_{k}}^{-1} \dot{s}_{i_{k-1}}^{-1} \ldots \dot{s}_{i_{m}}^{-1} x_{i_{m}}(h) \dot{s}_{i_{m}} \ldots \dot{s}_{i_{k-1}} \dot{s}_{i_{k}} \\
& =\dot{s}_{i_{k}}^{-1} \dot{s}_{i_{k-1}}^{-1} \ldots \dot{s}_{i_{m+1}}^{-1} y_{i_{m}}(-h) \dot{s}_{i_{m+1}} \ldots \dot{s}_{i_{k-1}} \dot{s}_{i_{k}} \in U_{A}^{-}
\end{aligned}
$$

(we have used 2.4(c), 2.5(b)). Hence $\dot{w}^{-1} u^{\prime} \dot{w} \in U_{A}^{-}$and $u^{\prime} \in U_{A}^{w}$. If $m \in[k+1, n]$ we have

$$
\dot{w}^{-1} r_{m} \dot{w}=\dot{s}_{i_{k+1}} \dot{s}_{i_{k+2}} \ldots \dot{s}_{i_{m-1}} x_{i_{m}}\left(h_{m}\right) \dot{s}_{i_{m-1}}^{-1} \ldots \dot{s}_{i_{k+2}}^{-1} \dot{s}_{i_{k+1}}^{-1} \in U_{A}
$$

(we have used 2.5(a)). Hence $\dot{w}^{-1} u^{\prime \prime} \dot{w} \in U_{A}$ and $u^{\prime \prime} \in{ }^{w} U_{A}$. If we assume that $u \in U_{A}^{w}$, then $u^{\prime \prime}=u^{\prime-1} u \in U_{A}^{w}$; hence $\dot{w}^{-1} u^{\prime \prime} \dot{w} \in U_{A}^{-}$. We also have $\dot{w}^{-1} u^{\prime \prime} \dot{w} \in U_{A}$. Since $U_{A} \cap U_{A}^{-}=\{1\}$ (by 2.3(b)) we have $\dot{w}^{-1} u^{\prime \prime} \dot{w}=1$ and $u^{\prime \prime}=1, u=u^{\prime}$. Conversely, if $u=u^{\prime}$, then, as we have seen, we have $u \in U_{A}^{w}$.

If we assume that $u \in{ }^{w} U_{A}$, then $u^{\prime}=u u^{\prime \prime-1} \in{ }^{w} U_{A}$; hence $\dot{w}^{-1} u^{\prime} \dot{w} \in U_{A}$. We also have $\dot{w}^{-1} u^{\prime} \dot{w} \in U_{A}^{-}$. Since $U_{A} \cap U_{A}^{-}=\{1\}$ (by $2.3(\mathrm{~b})$ ) we have $\dot{w}^{-1} u^{\prime} \dot{w}=1$ and $u^{\prime}=1, u=u^{\prime \prime}$. Conversely, if $u=u^{\prime \prime}$, then, as we have seen, we have $u \in{ }^{w} U_{A}$. Thus we have the following results:

(a) the restriction of the map 2.6(a) to $A^{k}$ (identified with

$$
\left\{\left(h_{1}, h_{2}, \ldots, h_{n}\right) \in A^{n} ; h_{k+1}=h_{k+2}=\cdots=h_{n}=0\right\}
$$

in the obvious way) defines a bijection $A^{k} \stackrel{\sim}{\longrightarrow} U_{A}^{w}$;

(b) the restriction of the map 2.6(a) to $A^{n-k}$ (identified with

$$
\left\{\left(h_{1}, h_{2}, \ldots, h_{n}\right) \in A^{n} ; h_{1}=h_{2}=\cdots=h_{k}=0\right\}
$$

in the obvious way) defines a bijection $A^{n-k} \stackrel{\sim}{\rightarrow}{ }^{w} U_{A}$.

Using (a),(b) and $\S 2.6$ we see also that

(c) multiplication defines a bijection $U_{A}^{w} \times{ }^{w} U_{A} \stackrel{\sim}{\rightarrow} U_{A}$. 
We show:

(d) multiplication in $G_{A}$ defines a bijection $\left(U_{A}^{w} \dot{w}\right) \times U_{A} \stackrel{\sim}{\rightarrow} U_{A} \dot{w} U_{A}$.

Using (c) we see that $U_{A} \dot{w} U_{A}=U_{A}^{w}\left({ }^{w} U_{A}\right) \dot{w} U_{A}=U_{A}^{w} \dot{w}\left(\dot{w}^{-1 w} U_{A} \dot{w}\right) U_{A} \subset U_{A}^{w} \dot{w} U_{A}$. Thus the map in (d) is surjective. Assume now that $u_{1}, u_{2} \in U_{A}^{w}$ and $u_{1}^{\prime}, u_{2}^{\prime} \in U_{A}$ satisfy $u_{1} \dot{w} u_{1}^{\prime}=u_{2} \dot{w} u_{2}^{\prime}$. Then $\dot{w}^{-1} u_{2}^{-1} u_{1} \dot{w}=u_{2}^{\prime} u_{1}^{\prime-1}$ is both in $U_{A}^{-}$and in $U_{A}$; hence by 2.3(b) it is 1 . Thus $u_{1}=u_{2}$ and $u_{1}^{\prime}=u_{2}^{\prime}$. Thus the map in (d) is injective. This proves (d).

Combining (d) with (a) and 2.6(a), we obtain a bijection

(e) $A^{k} \times A^{n} \stackrel{\sim}{\rightarrow} U_{A} \dot{w} U_{A}$

$$
\begin{aligned}
& \left(\left(h_{1}, \ldots, h_{k}\right),\left(h_{1}^{\prime}, \ldots, h_{n}^{\prime}\right)\right) \mapsto\left(x_{i_{1}}\left(h_{1}\right) \dot{s}_{i_{1}} x_{i_{2}}\left(h_{2}\right) \dot{s}_{i_{2}} \ldots x_{i_{k}}\left(h_{k}\right) \dot{s}_{i_{k}} \dot{w}^{-1}\right) \dot{w} \\
& \left(x_{i_{1}}\left(h_{1}^{\prime}\right) \dot{s}_{i_{1}} x_{i_{2}}\left(h_{2}^{\prime}\right) \dot{s}_{i_{2}} \ldots x_{i_{n}}\left(h_{n}^{\prime}\right) \dot{s}_{i_{n}} \dot{w}_{I}^{-1}\right) .
\end{aligned}
$$

We can reformulate (a) as follows.

(f) If $j_{1}, j_{2}, \ldots, j_{k}$ is a sequence in I such that $w=s_{j_{1}} \ldots s_{j_{k}}, l(w)=k$, then the map $A^{k} \rightarrow U_{A}^{w} \dot{w}$,

$$
\left(h_{1}, h_{2}, \ldots, h_{k}\right) \mapsto x_{i_{1}}\left(h_{1}\right) \dot{s}_{i_{1}} x_{i_{2}}\left(h_{2}\right) \dot{s}_{i_{2}} \ldots x_{i_{k}}\left(h_{k}\right) \dot{s}_{i_{k}}
$$

is a bijection.

For any sequence $w_{*}=\left(w_{1}, w_{2}, \ldots, w_{r}\right)$ in $W$ we set

$$
\begin{gathered}
U\left(w_{*}\right)=\left(U_{A}^{w_{1}} \dot{w}_{1}\right) \times\left(U_{A}^{w_{2}} \dot{w}_{2}\right) \times \cdots \times\left(U_{A}^{w_{r}} \dot{w}_{r}\right), \\
\dot{U}\left(w_{*}\right)=\left(U_{A} \dot{w}_{1} U_{A}\right) \times\left(U_{A} \dot{w}_{2} U_{A}\right) \times \cdots \times\left(U_{A} \dot{w}_{r} U_{A}\right) .
\end{gathered}
$$

We have an obvious inclusion $U\left(w_{*}\right) \subset \dot{U}\left(w_{*}\right)$. Let $\tilde{U}\left(w_{*}\right)$ be the set of orbits of the $U_{A}^{r-1}$-action

$$
\left(u_{1}, u_{2}, \ldots, u_{r-1}\right):\left(g_{1}, g_{2}, \ldots, g_{r}\right) \mapsto\left(g_{1} u_{1}^{-1}, u_{1} g_{2} u_{2}^{-1}, \ldots, u_{r-1} g_{r}\right)
$$

on $\dot{U}\left(w_{*}\right)$. Let $\kappa_{w_{*}}: \dot{U}\left(w_{*}\right) \rightarrow \tilde{U}\left(w_{*}\right)$ be the obvious surjective map; for $\left(g_{1}, \ldots, g_{r}\right) \in \dot{U}\left(w_{*}\right)$ we set $\left[g_{1}, \ldots, g_{r}\right]=\kappa_{w_{*}}\left(g_{1}, \ldots, g_{r}\right)$.

The following result is an immediate consequence of (f).

(g) Let $w \in W$ and let $w_{*}=\left(w_{1}, w_{2}, \ldots, w_{r}\right)$ be a sequence in $W$ such that $w=w_{1} w_{2} \ldots w_{r}, l(w)=l\left(w_{1}\right)+l\left(w_{2}\right)+\cdots+l\left(w_{r}\right)$. Then multiplication in $G_{A}$ defines a bijection $\phi_{w_{*}}: U\left(w_{*}\right) \stackrel{\sim}{\rightarrow} U_{A}^{w} \dot{w}$.

We show:

(h) Let $x, y \in W$ be such that $l(x y)=l(x)+l(y)$. Let $x_{*}=(x, y)$. Then multiplication in $G_{A}$ defines a bijection $\tilde{U}\left(x_{*}\right) \stackrel{\sim}{\rightarrow} U_{A} \dot{x} \dot{y} U_{A}$.

This follows from (d) and (g) since

$$
\tilde{U}\left(x_{*}\right) \cong\left(U_{A}^{x} \dot{x}\right) \times\left(U_{A} \dot{y} U_{A}\right)=\left(U_{A}^{x} \dot{x}\right) \times\left(U_{A}^{y} \dot{y} U_{A}\right)=U_{A}^{x y} \dot{x} \dot{y} U_{A} .
$$

Using (h) repeatedly we obtain:

(i) In the setup of $(g)$, multiplication in $G_{A}$ defines a bijection $\psi_{w_{*}}: \tilde{U}\left(w_{*}\right) \stackrel{\sim}{\rightarrow}$ $U_{A} \dot{w} U_{A}$. This bijection is compatible with the $U_{A} \times U_{A}$ actions

$\left(u, u^{\prime}\right):\left[g_{1}, g_{2}, \ldots, g_{r}\right] \mapsto\left[u g_{1}, g_{2}, \ldots, g_{r} u^{\prime-1}\right]$ on $\tilde{U}\left(w_{*}\right)$ and $\left(u, u^{\prime}\right): g \mapsto u g u^{\prime-1}$ on $U_{A} \dot{x} U_{A}$. 
2.8. Let $y_{*}=\left(y_{1}, y_{2}, \ldots, y_{t}\right)(t \geq 2)$ be a sequence in $W$. Let $\xi \in \tilde{U}\left(y_{*}\right)$. We show:

(a) for any $s \in[1, t-1]$ there exists a representative of $\xi$ in

$$
\left(U_{A}^{y_{1}} \dot{y}_{1}\right) \times \cdots \times\left(U_{A}^{y_{s-1}} \dot{y}_{s-1}\right) \times\left(U_{A} \dot{y}_{s} U_{A}\right) \times \cdots \times\left(U_{A} \dot{y}_{t} U_{A}\right) .
$$

We argue by induction on $s$. Let $\left(g_{1}, g_{2}, \ldots, g_{t}\right) \in \dot{U}\left(y_{*}\right)$ be a representative of $\xi$. We have $g_{1}=u \dot{y}_{1} u^{\prime}$, where $u \in U_{A}^{y_{1}}, u^{\prime} \in U_{A}$ (see $\left.2.7(\mathrm{~d})\right)$. Then $\left(u \dot{y}_{1}, u^{\prime} g_{2}, g_{3}, \ldots, g_{t}\right)$ represents $\xi$ and is as in (a) with $s=1$. Now assume that $s \geq 2$ and that $\left(g_{1}, g_{2}, \ldots, g_{t}\right)$ is a representative of $\xi$ as in (a) with $s$ replaced by $s-1$. We have $g_{s-1}=u \dot{y}_{s-1} u^{\prime}$ with $u \in U_{A}^{y_{s-1}}, u^{\prime} \in U_{A}$ (see 2.7(d)). Then

$$
\left(g_{1}, g_{2}, \ldots, g_{s-2}, u \dot{y}_{s-1}, u^{\prime} g_{s}, g_{s+1}, \ldots, g_{t}\right)
$$

is a representative of $\xi$ as in (a). This completes the inductive proof of (a).

We show:

(b) there exist unique elements $u_{1} \in U_{A}^{y_{1}}, \ldots, u_{t} \in U_{A}^{y_{t}}$ and $u \in U_{A}$ such that $\xi=\left[u_{1} \dot{y}_{1}, \ldots, u_{t-1} \dot{y}_{t-1}, u_{t} \dot{y}_{t} u\right]$.

The existence of these elements folows from (a) with $s=t-1$ and 2.7(d). We prove uniqueness. Assume that $u_{1}, u_{1}^{\prime} \in U_{A}^{y_{1}}, \ldots, u_{t}, u_{t}^{\prime} \in U_{A}^{y_{t}}$ and $u, u^{\prime} \in U_{A}$ are such that

$$
\left[u_{1} \dot{y}_{1}, \ldots, u_{t-1} \dot{y}_{t-1}, u_{t} \dot{y}_{t} u\right]=\left[u_{1}^{\prime} \dot{y}_{1}, \ldots, u_{t-1}^{\prime} \dot{y}_{t-1}, u_{t}^{\prime} \dot{y}_{t} u^{\prime}\right]=\xi .
$$

Then there exist $v_{1}, v_{2}, \ldots, v_{t-1}$ in $U_{A}$ such that

$$
\begin{aligned}
& u_{1}^{\prime} \dot{y}_{1}=u_{1} \dot{y}_{1} v_{1}, u_{2}^{\prime} \dot{y}_{2}=v_{1}^{-1} u_{2} \dot{y}_{2} v_{2}, \ldots, u_{t-1}^{\prime} \dot{y}_{t-1}=v_{t-2}^{-1} u_{t-1} \dot{y}_{t-1} v_{t-1}, \\
& u_{t}^{\prime} \dot{y}_{t} u^{\prime}=v_{t-1}^{-1} u_{t} \dot{y}_{t} u .
\end{aligned}
$$

The first of these equations implies (using 2.7(d)) that $v_{1}=1$ and $u_{1}^{\prime}=u_{1}$. Then the second equation becomes $u_{2}^{\prime} \dot{y}_{2}=u_{2} \dot{y}_{2} v_{2}$; using again $2.7(\mathrm{~d})$, we deduce that $v_{2}=1$ and $u_{2}^{\prime}=u_{2}$. Continuing in this way we get $v_{1}=\cdots=v_{t-1}=1$ and $u_{i}=u_{i}^{\prime}$ for $i \in[1, t-1]$. We then have $u_{t}^{\prime} \dot{y}_{t} u^{\prime}=u_{t} \dot{y}_{t} u$. Using $2.7(\mathrm{~d})$ we deduce $u_{t}^{\prime}=u_{t}$, $u^{\prime}=u$. This proves $(\mathrm{b})$.

We now define $\zeta: \tilde{U}\left(y_{*}\right) \rightarrow U_{A}$ by $\zeta(\xi)=u$, where $u$ is as in (b).

2.9. Let $\hat{W}$ be the braid monoid attached to $W$ and let $w \mapsto \hat{w}$ be the canonical map $W \rightarrow \hat{W}$. Let $x_{*}=\left(x_{1}, \ldots, x_{s}\right), y_{*}=\left(y_{1}, \ldots, y_{t}\right)$ be two sequences in $W$ such that $\hat{x}_{1} \ldots \hat{x}_{s}=\hat{y}_{1} \ldots \hat{y}_{t}$ in $\hat{W}$. We show:

(a) there exist bijections $H: U\left(x_{*}\right) \stackrel{\sim}{\rightarrow} U\left(y_{*}\right), \theta: \tilde{U}\left(x_{*}\right) \stackrel{\sim}{\rightarrow} \tilde{U}\left(y_{*}\right)$ such that $\kappa_{y_{*}} H=\theta \kappa_{x_{*}}$ and such that $\theta$ is compatible with the $U_{A} \times U_{A}$-actions (as in 2.7(i)). Applying $2.7(\mathrm{~g})$ and 2.7(i) with $w$ replaced by $x_{a}$ (resp. $\left.y_{b}\right)$ and $w_{1}, w_{2}, \ldots, w_{r}$ replaced by a sequence of simple reflections whose product is a reduced expression of $x_{a}$ (resp. $\left.y_{b}\right)$ we see that the general case is reduced to the case where $l\left(x_{1}\right)=$ $\cdots=l\left(x_{s}\right)=l\left(y_{1}\right)=\cdots=l\left(y_{t}\right)=1$; in this case we must have $s=t$. Since $\hat{x}_{1} \ldots \hat{x}_{s}=\hat{y}_{1} \ldots \hat{y}_{s}$ we can find a sequence $\mathbf{s}_{*}^{1}, \mathbf{s}_{*}^{2}, \ldots, \mathbf{s}_{*}^{m}$, where each $\mathbf{s}_{*}^{p}$ is a sequence in $S, \mathbf{s}_{*}^{1}=x_{*}, \mathbf{s}_{*}^{m}=y_{*}$ and for any $r \in[1, m-1], \mathbf{s}_{*}^{r+1}$ is related to $\mathbf{s}_{*}^{r}$ as follows:

(b) for some $i \neq j$ in $I$ and some $e$ such that $[e+1, e+u] \subset[1, s]\left(n_{i j}=u\right)$ we have

$$
\begin{aligned}
& \mathbf{s}_{c}^{r}=\mathbf{s}_{c}^{r+1} \text { if } c \in[1, s]-[e+1, e+u], \\
& \left(\mathbf{s}_{e+1}^{r}, \mathbf{s}_{e+2}^{r}, \ldots, \mathbf{s}_{e+u}^{r}\right)=s_{*}:=\left(s_{i}, s_{j}, s_{i}, \ldots\right), \\
& \left(\mathbf{s}_{e+1}^{r+1}, \mathbf{s}_{e+2}^{r+1}, \ldots, \mathbf{s}_{e+u}^{r+1}\right)=s_{*}^{\prime}:=\left(s_{j}, s_{i}, s_{j}, \ldots\right) .
\end{aligned}
$$

Note that each pair $\mathbf{s}_{*}^{r}, \mathbf{s}_{*}^{r+1}$ satisfies the same assumptions as the pair $x_{*}, y_{*}$. If (a) can be proved for each $\mathbf{s}^{r}, \mathbf{s}^{r+1}$, then (a) would follow also for $x_{*}, y_{*}$ (by taking appropriate compositions of maps such as $H$ or maps such as $\theta$ ). It is then enough 
to prove (a) assuming that $x_{*}=\mathbf{s}_{*}^{r}, y_{*}=\mathbf{s}_{*}^{r+1}$ are as in (b). Let $w=s_{i} s_{j} s_{i} \ldots=$ $s_{j} s_{i} s_{j} \ldots$ ( $u$ factors $)$. Let

$$
x_{*}^{\prime}=\left(x_{1}, \ldots, x_{e}\right)=\left(y_{1}, \ldots, y_{e}\right), \quad x_{*}^{\prime \prime}=\left(x_{e+u+1}, \ldots, x_{s}\right)=\left(x_{e+u+1}, \ldots, x_{s}\right) .
$$

Then

$$
U\left(x_{*}\right)=U\left(x_{*}^{\prime}\right) \times U\left(s_{*}\right) \times U\left(x_{*}^{\prime \prime}\right), \quad U\left(y_{*}\right)=U\left(x_{*}^{\prime}\right) \times U\left(s_{*}^{\prime}\right) \times U\left(x_{*}^{\prime \prime}\right) .
$$

Now $\tilde{U}\left(x_{*}\right)$ (resp. $\tilde{U}\left(y_{*}\right)$ ) is the set of orbits for the action of a subgroup of $U_{A} \times U_{A}$ on $\tilde{U}\left(x_{*}^{\prime}\right) \times \tilde{U}\left(s_{*}\right) \times \tilde{U}\left(x_{*}^{\prime \prime}\right)\left(\operatorname{resp} . \tilde{U}\left(x_{*}^{\prime}\right) \times \tilde{U}\left(s_{*}^{\prime}\right) \times \tilde{U}\left(x_{*}^{\prime \prime}\right)\right)$ given by

$$
\left(u, u^{\prime}\right):\left(\xi, \xi^{\prime}, \xi^{\prime \prime}\right) \mapsto\left((1, u) \xi,\left(u, u^{\prime}\right) \xi^{\prime},\left(u^{\prime}, 1\right) \xi^{\prime \prime}\right)
$$

(the subgroup is $U_{A} \times U_{A}$ if $x_{*}^{\prime}, x_{*}^{\prime \prime}$ are nonempty, is $\{1\} \times U_{A}$ if $x_{*}^{\prime}$ is empty and $x_{*}^{\prime \prime}$ is nonempty, is $U_{A} \times\{1\}$ if $x_{*}^{\prime}$ is nonempty and $x_{*}^{\prime \prime}$ is empty, is $\{1\} \times\{1\}$ if $x_{*}^{\prime}$ and $x_{*}^{\prime \prime}$ are empty). Let $\mathcal{T}$ be the set of orbits of the same subgroup of $U_{A} \times U_{A}$ on $\tilde{U}\left(x_{*}^{\prime}\right) \times\left(U_{A} \dot{w} U_{A}\right) \times \tilde{U}\left(x_{*}^{\prime \prime}\right)$ for the action given by the same formulas as above. We have a diagram

$$
U\left(x_{*}\right) \stackrel{h}{\rightarrow} U\left(x_{*}^{\prime}\right) \times\left(U_{A}^{w} \dot{w}\right) \times U\left(x_{*}^{\prime \prime}\right) \stackrel{h^{\prime}}{\longleftarrow} U\left(y_{*}\right)
$$

where

$$
h\left(\xi, \xi^{\prime}, \xi^{\prime \prime}\right)=\left(\xi, \phi_{s_{*}}\left(\xi^{\prime}\right), \xi^{\prime \prime}\right), \quad h^{\prime}\left(\xi, \xi^{\prime}, \xi^{\prime \prime}\right)=\left(\xi, \phi_{s_{*}^{\prime}}\left(\xi^{\prime}\right), \xi^{\prime \prime}\right)
$$

(with $\phi_{s_{*}}, \phi_{s_{*}^{\prime}}$ as in $2.7(\mathrm{~g})$. Note that $h, h^{\prime}$ are bijections. We set $H=h^{\prime-1} h$. We have a diagram

$$
\tilde{U}\left(x_{*}\right) \stackrel{\tilde{h}}{\rightarrow} \mathcal{T} \stackrel{\tilde{h}^{\prime}}{\longleftarrow} \tilde{U}\left(y_{*}\right)
$$

where

$$
\tilde{h}\left(\xi, \xi^{\prime}, \xi^{\prime \prime}\right)=\left(\xi, \psi_{s_{*}}\left(\xi^{\prime}\right), \xi^{\prime \prime}\right), \quad \tilde{h}^{\prime}\left(\xi, \xi^{\prime}, \xi^{\prime \prime}\right)=\left(\xi, \psi_{s_{*}^{\prime}}\left(\xi^{\prime}\right), \xi^{\prime \prime}\right)
$$

(with $\psi_{s_{*}}, \psi_{s_{*}^{\prime}}$ as in $2.7(\mathrm{i})$ ). Note that $\tilde{h}, \tilde{h}^{\prime}$ are bijections (they are well defined by $2.7(\mathrm{i}))$. We set $\theta=\left(\tilde{h}^{\prime}\right)^{-1} \tilde{h}$. It is clear that $H, \theta$ satisfy the requirements of (a). This proves (a).

2.10. Let $\delta$ be an automorphism of $\mathcal{R}$, that is, a triple consisting of automorphisms $\delta: Y \rightarrow Y, \delta: X \rightarrow X$ and a bijection $\delta: I \rightarrow I$ such that $\langle\delta(y), \delta(x)\rangle=\langle y, x\rangle$ for $y \in Y, x \in X, \delta$ is compatible with the imbeddings $I \rightarrow Y, I \rightarrow X$ and $\delta(i) \cdot \delta(j)=i \cdot j$ for $i, j \in I$. There is a unique group automorphism of $W, w \mapsto \delta(w)$ such that $\delta\left(s_{i}\right)=s_{\delta(i)}$ for all $i \in I$. There is a unique algebra automorphism (preserving 1) of $\mathbf{f}_{A}, x \mapsto \delta(x)$, such that $\delta\left(\theta_{i}^{(c)}\right)=\theta_{\delta(i)}^{(c)}$ for all $i \in I, c \in \mathbf{N}$. We have $\delta(\mathbf{B})=\mathbf{B}$. There is a unique algebra automorphism of $\dot{\mathbf{U}}_{A}, u \mapsto \delta(u)$, such that $\delta\left(b^{-} 1_{\zeta} b^{\prime+}\right)=\delta(b)^{-} 1_{\delta(\zeta)} \delta\left(b^{\prime}\right)^{+}$for all $b \in \mathbf{B}, b^{\prime} \in \mathbf{B}, \zeta \in X$. We have $\delta(\dot{\mathbf{B}})=\dot{\mathbf{B}}$. There is a unique algebra automorphism (preserving 1) of $\hat{\mathbf{U}}_{A}, u \mapsto \delta(u)$ such that $\delta\left(\sum_{a \in \dot{\mathbf{B}}} n_{a} a\right)=\sum_{a \in \dot{\mathbf{B}}} n_{a} \delta(a)$ for all functions $\dot{\mathbf{B}} \rightarrow A, a \mapsto n_{a}$. This automorphism restricts to a group automorphism $G_{A} \rightarrow G_{A}$ denoted again by $\delta$ and to an automorphism of $U_{A}$. For any $i \in I, h \in H$ we have $\delta\left(x_{i}(h)\right)=x_{\delta(i)}(h)$, $\delta\left(\dot{s}_{i}\right)=\dot{s}_{\delta(i)}$. 
2.11. Let $A \in \mathcal{C}, A^{\prime} \in \mathcal{C}$ and let $\chi: A \rightarrow A^{\prime}$ be a homomorphism of rings preserving 1. There is a unique ring homomorphism (preserving 1) $\hat{\mathbf{U}}_{A} \rightarrow \hat{\mathbf{U}}_{A^{\prime}}, u \mapsto \chi(u)$ such that $\chi\left(\sum_{a \in \dot{\mathbf{B}}} n_{a} a\right)=\sum_{a \in \dot{\mathbf{B}}} \chi\left(n_{a}\right) a$ for all functions $\dot{\mathbf{B}} \rightarrow A, a \mapsto n_{a}$. This restricts to a group homomorphism $G_{A} \rightarrow G_{A^{\prime}}$ denoted again by $\chi$ and to a group homomorphism $U_{A} \rightarrow U_{A^{\prime}}$. For any $i \in I, h \in A$ we have $\chi\left(x_{i}(h)\right)=x_{i}(\chi(h))$, $\chi\left(\dot{s}_{i}\right)=\dot{s}_{i}$.

\section{THE MAIN RESULTS}

3.1. In this section $A \in \mathcal{C}$ is fixed unless otherwise specified. We also fix an automorphism $\delta$ of $\mathcal{R}$ as in $\S 2.10$ and a ring automorphism $\chi$ of $A$ preserving 1 . There are induced group automorphisms of $G_{A}$ denoted again by $\delta, \chi$ (see $\S 2.10$, $\S 2.11)$. These automorphisms commute; we set $\pi=\delta \chi=\chi \delta: G_{A} \rightarrow G_{A}$; note that $\pi$ maps $U_{A}$ onto itself.

Two elements $w, w^{\prime}$ of $W$ are said to be $\delta$-conjugate if $w^{\prime}=y^{-1} w \delta(y)$ for some $y \in W$. The relation of $\delta$-conjugacy is an equivalence relation on $W$; the equivalence classes are said to be $\delta$-conjugacy classes. A $\delta$-conjugacy class $C$ in $W$ (or an element of it) is said to be $\delta$-elliptic if $C \cap W_{J}=\emptyset$ for any $J \varsubsetneqq I, \delta(J)=J$. Let $C$ be a $\delta$-elliptic $\delta$-conjugacy class in $W$. Let $C_{\text {min }}$ be the set of elements of minimal length of $C$.

For any $w \in W$ we define a map

$$
\Xi_{A}^{w}: U_{A} \times\left(U_{A}^{w} \dot{w}\right) \rightarrow U_{A} \dot{w} U_{A}
$$

by $(u, z) \mapsto u z \pi(u)^{-1}$.

3.2. In this subsection we assume that $x, y \in W$ are such that $l(x \delta(y))=l(x)+$ $l(y)=l(y x)$. We show:

$(*) \Xi_{A}^{x \delta(y)}$ is injective if and only if $\Xi_{A}^{y x}$ is injective. Let $x_{*}=(x, \delta(y)), x_{*}^{\prime}=$ $(y, x)$.

In the following proof we write $U, U^{x}, U^{y}, U^{\delta(y)}$ instead of $U_{A}, U_{A}^{x}, U_{A}^{y}, U_{A}^{\delta(y)}$. Now $\Xi_{A}^{x \delta(y)}$ can be identified with

$$
U \times\left(U^{x} \dot{x}\right) \times\left(U^{\delta(y)} \delta(\dot{y})\right) \rightarrow \tilde{U}\left(x_{*}\right), \quad\left(u, z, z^{\prime}\right) \mapsto\left[u z, z^{\prime} \pi(u)^{-1}\right]
$$

and $\Xi_{A}^{y x}$ can be identified with

$$
U \times\left(U^{y} \dot{y}\right) \times\left(U^{x} \dot{x}\right) \rightarrow \tilde{U}\left(x_{*}^{\prime}\right), \quad\left(u, z^{\prime}, z\right) \mapsto\left[u z^{\prime}, z \pi(u)^{-1}\right] .
$$

(We use $2.7(\mathrm{~g}), 2.7(\mathrm{i})$.) The condition that $\Xi_{A}^{x \delta(y)}$ is injective can be stated as follows:

(a) if $u \in U, z_{1}, z_{2} \in U^{x} \dot{x}, z_{3}, z_{4} \in U^{\delta(y)} \delta(\dot{y})$ satisfy $u z_{1} v^{-1}=z_{2}, v z_{3} \pi(u)^{-1}=z_{4}$ for some $v \in U$, then $u=1$.

The condition that $\Xi_{A}^{y x}$ is injective can be stated as follows:

(b) if $u^{\prime} \in U, z_{1}^{\prime}, z_{2}^{\prime} \in U^{x} \dot{x}, z_{3}^{\prime}, z_{4}^{\prime} \in U^{y} \dot{y}$ satisfy $u^{\prime} z_{3}^{\prime} v^{\prime-1}=z_{4}^{\prime}, v^{\prime} z_{1}^{\prime} \pi\left(u^{\prime}\right)^{-1}=z_{2}^{\prime}$ for some $v^{\prime} \in U$, then $u^{\prime}=1$.

Assume that (a) holds and that the hypothesis of (b) holds. We have $v^{\prime} z_{1}^{\prime} \pi\left(u^{\prime}\right)^{-1}=$ $z_{2}^{\prime}, \pi\left(u^{\prime}\right) \pi\left(z_{3}^{\prime}\right) \pi\left(v^{\prime}\right)^{-1}=\pi\left(z_{4}^{\prime}\right)$ and $\pi\left(z_{3}^{\prime}\right), \pi\left(z_{4}^{\prime}\right) \in U^{\delta(y)} \delta(\dot{y})$. Applying (a) with $v=\pi\left(u^{\prime}\right), u=v^{\prime}$ we obtain $v^{\prime}=1$. Then $\pi\left(u^{\prime}\right)=z_{2}^{\prime-1} z_{1}^{\prime} \in \dot{x}^{-1} U^{x} \dot{x} \subset U^{-}$. But $U \cap U^{-}=\{1\}$ (see 2.3(b)); hence $u^{\prime}=1$. Thus the conclusion of (b) holds.

Next we assume that (b) holds and that the hypothesis of (a) holds. We have $\pi^{-1}(v) \pi^{-1}\left(z_{3}\right) u^{-1}=\pi^{-1}\left(z_{4}\right)$ and $\pi^{-1}\left(z_{3}\right), \pi^{-1}\left(z_{4}\right) \in U^{y} \dot{y}$. Applying (b) with 
$v^{\prime}=u, u^{\prime}=\pi^{-1}(v)$ we obtain $v=1$. Then $\pi(u)=z_{4}^{-1} z_{3} \in \dot{y}^{-1} U^{y} \dot{y} \subset U^{-}$. But $U \cap U^{-}=\{1\}$; hence $u=1$. Thus the conclusion of (a) holds. We see that (a) holds if and only if (b) holds. This proves $(*)$.

3.3. In this subsection we assume that $x, y \in W$ are such that $l(y x)=l(x)+l(y)$ and we write $U, U^{x}, U^{y}, U^{y x}$ instead of $U_{A}, U_{A}^{x}, U_{A}^{y}, U_{A}^{y x}$. We have a commutative diagram

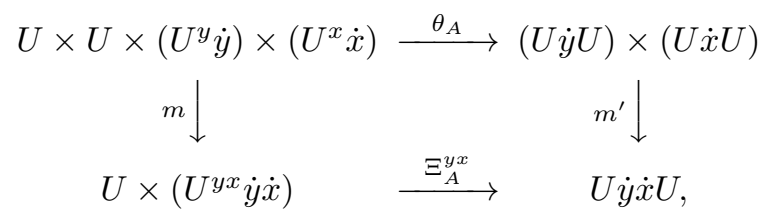

where

$\theta_{A}\left(u, u^{\prime}, g, g^{\prime}\right)=\left(u g u^{\prime-1}, u^{\prime} g^{\prime} \pi(u)^{-1}\right), \quad m\left(u, u^{\prime}, g, g^{\prime}\right)=\left(u, g g^{\prime}\right), \quad m^{\prime}\left(g, g^{\prime}\right)=g g^{\prime}$.

We show:

(a) the sets

$$
\begin{gathered}
S^{\prime}=\left\{\Xi^{\prime}: U \dot{y} \dot{x} U \rightarrow U \times\left(U^{y x} \dot{y} \dot{x}\right) ; \Xi_{A}^{y x} \Xi^{\prime}=1\right\} \\
S^{\prime \prime}=\left\{Z:(U \dot{y} U) \times(U \dot{x} U) \rightarrow U \times U \times\left(U^{y} \dot{y}\right) \times\left(U^{x} \dot{x}\right) ; \theta_{A} Z=1\right\}
\end{gathered}
$$

are in natural bijection.

Let $\Xi^{\prime} \in S^{\prime}$. We define $Z$ as follows. Let $\left(z, z^{\prime}\right) \in(U \dot{y} U) \times(U \dot{x} U)$. We have $\Xi^{\prime}\left(z z^{\prime}\right)=\left(u, g g^{\prime}\right)$, where $u \in U, g \in U^{y} \dot{y}, g^{\prime} \in U^{x} \dot{x}$ are uniquely defined. Since $u g \in U \dot{y} U$ we can write uniquely $u g=z_{0} u^{\prime}$, where $z_{0} \in U^{y} \dot{y}, u^{\prime} \in U$ (see $\left.2.7(\mathrm{~d})\right)$. We set $Z\left(z, z^{\prime}\right)=\left(u, u^{\prime}, g, g^{\prime}\right)$. This defines the map $Z$. We have

$$
z z^{\prime}=\Xi_{A}^{y x}\left(\Xi^{\prime}\left(z z^{\prime}\right)\right)=u g g^{\prime} \pi(u)^{-1} .
$$

The equality $z z^{\prime}=(u g)\left(g^{\prime} \pi(u)^{-1}\right)$ and $2.7(\mathrm{~h})$ imply that $u g v^{-1}=z, v g^{\prime} \pi(u)^{-1}=z^{\prime}$ for a well-defined $v \in U$. We have $u g=z v=z_{0} u^{\prime}$. From $z v=z_{0} u^{\prime}$ and 2.7(d) we see that $u^{\prime}=v$. Thus $u g u^{\prime-1}=z, u^{\prime} g^{\prime} \pi(u)^{-1}=z^{\prime}$. Hence

$$
\theta_{A}\left(Z\left(z, z^{\prime}\right)\right)=\theta_{A}\left(u, u^{\prime}, g, g^{\prime}\right)=\left(u g u^{\prime-1}, u^{\prime} g^{\prime} \pi(u)^{-1}\right)=\left(z, z^{\prime}\right) .
$$

Thus $\theta_{A} Z=1$ and $Z \in S^{\prime \prime}$.

Conversely, let $Z \in S^{\prime \prime}$. We define $\Xi^{\prime}$ as follows. Let $h \in U \dot{y} \dot{x} U$. Using $2.7(\mathrm{~h})$ and $2.7(\mathrm{~d})$ we can write uniquely $h=z z^{\prime}$ where $z \in U^{y} \dot{y}, z^{\prime} \in U \dot{x} U$. We set $\Xi^{\prime}(h)=m\left(Z\left(z, z^{\prime}\right)\right)$. We have

$$
\Xi_{A}^{y x}\left(\Xi^{\prime}(h)\right)=\Xi_{A}^{y x}\left(m\left(Z\left(z, z^{\prime}\right)\right)=m^{\prime}\left(\theta_{A}\left(Z\left(z, z^{\prime}\right)\right)=m^{\prime}\left(z, z^{\prime}\right)=z z^{\prime}=h .\right.\right.
$$

Thus $\Xi_{A}^{y x} \Xi^{\prime}=1$ and $\Xi^{\prime} \in S^{\prime}$.

It is easy to check that the maps $S^{\prime} \rightarrow S^{\prime \prime}, S^{\prime \prime} \rightarrow S^{\prime}$ defined above are inverse to each other. This proves (a).

3.4. In this subsection we assume that $x, y \in W$ are such that

$$
l(x \delta(y))=l(x)+l(y)=l(y x)
$$

and we write $U, U^{x}, U^{y}, U^{\delta(y)}, U^{y x}, U^{x \delta(y)}$ instead of $U_{A}, U_{A}^{x}, U_{A}^{y}, U_{A}^{\delta(y)}, U_{A}^{y x}, U_{A}^{x \delta(y)}$. 
We show:

(a) the sets

$$
\begin{gathered}
S_{1}^{\prime}=\left\{\Xi_{1}^{\prime}: U \dot{y} \dot{x} U \rightarrow U \times\left(U^{y x} \dot{y} \dot{x}\right) ; \Xi_{A}^{y x} \Xi_{1}^{\prime}=1\right\}, \\
S_{2}^{\prime}=\left\{\Xi_{2}^{\prime}: U \dot{x} \delta(\dot{y}) U \rightarrow U \times\left(U^{x \delta(y)} \dot{x} \delta(\dot{y})\right) ; \Xi_{A}^{y x} \Xi_{2}^{\prime}=1\right\}
\end{gathered}
$$

are in natural bijection.

Define

$$
\theta: U \times U \times\left(U^{y} \dot{y}\right) \times\left(U^{x} \dot{x}\right) \rightarrow(U \dot{y} U) \times(U \dot{x} U)
$$

and

$$
\theta^{\prime}: U \times U \times\left(U^{x} \dot{x}\right) \times\left(U^{\delta(y)} \delta(\dot{y})\right) \rightarrow(U \dot{x} U) \times(U \delta(\dot{y}) U)
$$

by $\left(u, u^{\prime}, g, g^{\prime}\right) \mapsto\left(u g u^{\prime-1}, u^{\prime} g^{\prime} \pi(u)^{-1}\right)$. In view of $\S 3.3$ applied to $x, y$ and also to $\delta(y), x$ we see that to prove (a), it is enough to show:

(b) the sets

$$
\begin{gathered}
S_{1}^{\prime \prime}=\left\{Z:(U \dot{y} U) \times(U \dot{x} U) \rightarrow U \times U \times\left(U^{y} \dot{y}\right) \times\left(U^{x} \dot{x}\right) ; \theta Z=1\right\}, \\
S_{2}^{\prime \prime}=\left\{Z^{\prime}:(U \dot{x} U) \times(U \delta(\dot{y}) U) \rightarrow U \times U \times\left(U^{x} \dot{x}\right) \times\left(U^{\delta(y)} \delta(\dot{y})\right) ; \theta^{\prime} Z^{\prime}=1\right\}
\end{gathered}
$$

are in natural bijection.

Now (b) follows from the commutative diagram

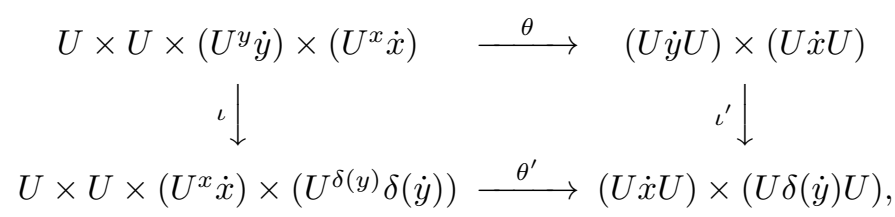

where $\iota\left(u, u^{\prime}, g, g^{\prime}\right)=\left(u^{\prime}, \pi(u), g^{\prime}, \pi(g)\right)$ and $\iota^{\prime}\left(g, g^{\prime}\right)=\left(g^{\prime}, \pi(g)\right)$ are bijections. This proves (a).

3.5. We show:

(a) if $\Xi_{A}^{w}$ is injective for some $w \in C_{\text {min }}$, then $\Xi_{A}^{w}$ is injective for any $w \in C_{\text {min }}$. For any $w, w^{\prime}$ in $C_{\text {min }}$ there exists a sequence $w=w_{1}, w_{2}, \ldots, w_{r}=w^{\prime}$ in $C_{\text {min }}$ such that for any $h \in[1, r-1]$ we have either $w_{h}=x \delta(y), w_{h+1}=y x$ for some $x, y$ as in $\S 3.2$ or $w_{h+1}=x \delta(y), w_{h}=y x$ for some $x, y$ as in $\S 3.2$. (See GP, GKP, He. .) Now (a) follows by applying $3.2(*)$ several times.

Now assume that for some $w^{\prime} \in C_{\text {min }}$, we are given $\Xi^{\prime}: U_{A} \dot{w}^{\prime} U_{A} \rightarrow U_{A} \times$ $\left(U_{A}^{w^{\prime}} \dot{w}^{\prime}\right)$ such that $\Xi_{A}^{w^{\prime}} \Xi^{\prime}=1$. Let $w \in C_{\min }$. We show how to construct a map $\Xi^{\prime \prime}: U_{A} \dot{w} U_{A} \rightarrow U_{A} \times\left(U_{A}^{w} \dot{w}\right)$ such that $\Xi_{A}^{w} \Xi^{\prime \prime}=1$. We choose a sequence $w=w_{1}, w_{2}, \ldots, w_{r}=w^{\prime}$ in $C_{\min }$ as in the proof of (a). We define a sequence of maps $\Xi_{i}^{\prime}: U_{A} \dot{w}_{i} U_{A} \rightarrow U_{A} \times\left(U_{A}^{w_{i}} \dot{w}_{i}\right)(i \in[1, r])$ such that $\Xi_{A}^{w_{i}} \Xi_{i}^{\prime}=1$ by induction on $i$ as follows. We set $\Xi_{1}^{\prime}=\Xi^{\prime}$. Assuming that $\Xi_{i}^{\prime}$ is defined for some $i \in[1, r-1]$ we define $\Xi_{i+1}^{\prime}$ so that $\Xi_{i}^{\prime}, \Xi_{i+1}^{\prime}$ correspond to each other under a bijection as in $\S 3.4$. Then the map $\Xi^{\prime \prime}:=\Xi_{r}^{\prime}$ satisfies our requirement. In particular, we see that: $C_{\text {min }}$.

(b) if $\Xi_{A}^{w}$ is surjective for some $w \in C_{\text {min }}$, then $\Xi_{A}^{w}$ is surjective for any $w \in$

Theorem 3.6. Recall that $A \in \mathcal{C}$. Let $C$ be a $\delta$-elliptic $\delta$-conjugacy class in $W$ and let $w \in C_{\min }$. Then:

(i) $\Xi_{A}^{w}$ is injective;

(ii) if $\chi=1$, then $\Xi_{A}^{w}$ is bijective; 
(iii) if $A$ is a field, $\chi$ has finite order $m$ and the fixed point field $A^{\chi}$ is perfect, then $\Xi_{A}^{w}$ is bijective;

(iv) if $A$ is an algebraic closure of a finite field $F_{q}$ and $\chi(x)=x^{q}$ for all $x \in A$, then $\Xi_{A}^{w}$ is bijective;

(v) if $A$ is finite and $\chi$ is arbitrary, then $\Xi_{A}^{w}$ is bijective.

The proof will occupy $\S \S 3.7-3.10$. If $W=\{1\}$ the result is trivial. Hence we can assume that $W \neq\{1\}$. We set $N=l(w)+l\left(w_{I}\right)$ for some/any $w \in C$.

3.7. Let $e$ be the smallest integer $\geq 1$ such that $\delta^{e}=1$ on $W$ and $w \delta(w) \delta^{2}(w) \ldots$ $\delta^{e-1}(w)=1$ for some (or equivalently any) $w \in C$. According to GM], GKP, [He, we can find an element $w^{\prime} \in C_{\min }$ such that $\hat{w}^{\prime} \widehat{\delta\left(w^{\prime}\right)} \ldots \widehat{\delta^{e-1}\left(w^{\prime}\right)}=\hat{y}_{1} \hat{y}_{2} \ldots \hat{y}_{t}$ (in the braid monoid $\hat{W}$ ), where $y_{*}=\left(y_{1}, y_{2}, \ldots, y_{t}\right)$ is a sequence in $W$ such that $y_{1}=w_{I}$. [Note added 10.4.2011. A proof of the existence of $w^{\prime}$ which does not rely on computer calculations has meanwhile been given in X. He and S. Nie, Minimal length elements of finite Coxeter groups, arxiv:1108.0282.]

Since $W \neq\{1\}$ we have $t \geq 2$. From now until the end of $\S 3.9$ we assume that $w=w^{\prime}$. Let $x_{*}=\left(w, \delta(w), \ldots, \delta^{e-1}(w)\right)$ and let $H: U\left(x_{*}\right) \stackrel{\sim}{\rightarrow} U\left(y_{*}\right), \theta: U\left(x_{*}\right) \stackrel{\sim}{\longrightarrow}$ $U\left(y_{*}\right)$ be as in 2.9(a). Let $z \in U_{A} \dot{w} U_{A}$. Then $\left[z, \pi(z), \ldots, \pi^{e-1}(z)\right] \in \tilde{U}\left(x_{*}\right)$; hence $\theta\left[z, \pi(z), \ldots, \pi^{e-1}(z)\right] \in \tilde{U}\left(y_{*}\right)$. We set $u=\zeta\left(\theta\left[z, \pi(z), \ldots, \pi^{e-1}(z)\right]\right) \in U_{A}$, where $\zeta: U\left(y_{*}\right) \rightarrow U$ is as in $\S 2.8$. We can write uniquely $\pi^{-e}(u) z \pi^{-e+1}(u)^{-1}=z^{\prime} u^{\prime}$, where $z^{\prime} \in U_{A}^{w} \dot{w}, u^{\prime} \in U_{A}$ (see $\left.2.7(\mathrm{~d})\right)$. We set $\Xi_{A}^{\prime}(z)=\left(\pi^{-e}(u)^{-1}, z^{\prime}\right) \in U_{A} \times$ $\left(U_{A}^{w} \dot{w}\right)$. Thus we have a map

$$
\Xi_{A}^{\prime}: U_{A} \dot{w} U_{A} \rightarrow U_{A} \times\left(U_{A}^{w} \dot{w}\right) .
$$

We show:

(a) $\Xi_{A}^{\prime} \Xi_{A}^{w}$ is the identity map of $U_{A} \times\left(U_{A}^{w} \dot{w}\right)$ into itself.

Let $\left(u_{1}, z_{1}\right) \in U_{A} \times\left(U_{A}^{w} \dot{w}\right)$. Let $z=u_{1} z_{1} \pi\left(u_{1}\right)^{-1}$. We must show that $\Xi_{A}^{\prime}(z)=$ $\left(u_{1}, z_{1}\right)$. Let $\xi=\theta\left[z, \pi(z), \ldots, \pi^{e-1}(z)\right] \in \tilde{U}\left(y_{*}\right)$. By 2.8(b) we have $\xi=$ $\left(1, u^{-1}\right)\left[h_{1}, \ldots, h_{t}\right]$ with $\left(h_{1}, \ldots, h_{t}\right) \in U\left(y_{*}\right), u \in U_{A}$ uniquely determined; moreover from the definitions we have $u=\zeta(\xi)$. We have

$$
\begin{aligned}
& {\left[z, \pi(z), \ldots, \pi^{e-1}(z)\right]} \\
& =\left[u_{1} z_{1} \pi\left(u_{1}\right)^{-1}, \pi\left(u_{1}\right) \pi\left(z_{1}\right) \pi^{2}\left(u_{1}\right)^{-1}, \ldots, \pi^{e-1}\left(u_{1}\right) \pi^{e-1}\left(z_{1}\right) \pi^{e}\left(u_{1}\right)^{-1}\right] \\
& =\left[u_{1} z_{1}, \pi\left(z_{1}\right), \ldots, \pi^{e-2}\left(z_{1}\right), \pi^{e-1}\left(z_{1}\right) \pi^{e}\left(u_{1}\right)^{-1}\right] \\
& =\left(u_{1}, \pi^{e}\left(u_{1}\right)\right)\left[z_{1}, \pi\left(z_{1}\right), \ldots, \pi^{e-2}\left(z_{1}\right), \pi^{e-1}\left(z_{1}\right)\right] \in \tilde{U}\left(x_{*}\right) ;
\end{aligned}
$$

hence

$$
\begin{aligned}
& \xi=\theta\left(\left(u_{1}, \pi^{e}\left(u_{1}\right)\right)\left[z_{1}, \pi\left(z_{1}\right), \ldots, \pi^{e-2}\left(z_{1}\right), \pi^{e-1}\left(z_{1}\right)\right]\right) \\
& =\left(u_{1}, \pi^{e}\left(u_{1}\right)\right) \theta\left(\left[z_{1}, \pi\left(z_{1}\right), \ldots, \pi^{e-2}\left(z_{1}\right), \pi^{e-1}\left(z_{1}\right)\right]\right) \\
& =\left(u_{1}, \pi^{e}\left(u_{1}\right)\right)\left[a_{1}, \ldots, a_{t}\right]
\end{aligned}
$$

where $\left(a_{1}, \ldots, a_{t}\right)=H\left(z_{1}, \pi\left(z_{1}\right), \ldots, \pi^{e-2}\left(z_{1}\right), \pi^{e-1}\left(z_{1}\right)\right) \in U\left(y_{*}\right)$. Thus we have

$$
\left(1, u^{-1}\right)\left[h_{1}, \ldots, h_{t}\right]=\left(u_{1}, \pi^{e}\left(u_{1}\right)\right)\left[a_{1}, \ldots, a_{t}\right] \in \tilde{U}\left(y_{*}\right)
$$

and

$$
\left[h_{1}, \ldots, h_{t}\right]=\left[u_{1} a_{1}, a_{2}, \ldots, a_{t-1}, a_{t} \pi^{e}\left(u_{1}\right)^{-1} u^{-1}\right] \in \tilde{U}\left(y_{*}\right)
$$


Note that $h_{i} \in U_{A}^{y_{i}} \dot{y}_{i}$ for $i \in[1, t], a_{i} \in U_{A}^{y_{i}} \dot{y}_{i}$ for $i \in[2, t]$ and $u_{1} a_{1} \in U_{A}^{y_{1}} \dot{y}_{1}$ (we use that $\left.y_{1}=w_{I}, U_{A}^{w_{I}}=U_{A}\right)$. Using the uniqueness part of 2.8(b) we deduce that $\pi^{e}\left(u_{1}\right)^{-1} u^{-1}=1$; hence $\pi^{-e}(u)=u_{1}^{-1}$. Then we have

$$
\pi^{-e}(u) z \pi^{-e+1}(u)^{-1}=u_{1}^{-1} z \pi\left(u_{1}\right)=z_{1} \in U_{A}^{w} \dot{w} .
$$

Using the definition we have $\Xi_{A}^{\prime}(z)=\left(\pi^{-e}(u)^{-1}, z_{1}\right)=\left(u_{1}, z_{1}\right)$. This proves (a).

3.8. If $w$ is as in $\S 3.7$, then from $3.7(\mathrm{a})$ we see that $\Xi_{A}^{w}$ is injective. This proves 3.6(i) for this $w$.

3.9. Let $w$ be as in $\S 3.7$. Let $N$ be as in $\S 3.6$. We identify $A^{N}=U_{A} \times\left(U_{A}^{w} \dot{w}\right)$ as in 2.6(a), 2.7(e) and $A^{N}=U_{A} \dot{w} U_{A}$ as in 2.7(f). Then $\Xi_{A}^{w}$ and $\Xi_{A}^{\prime}$ become maps $f_{A}: A^{N} \rightarrow A^{N}, f_{A}^{\prime}: A^{N} \rightarrow A^{N}$ such that $f_{A}^{\prime} f_{A}=1$. Assuming that $\chi=1$ and $\delta$ is fixed, we see from the definitions that $\left(f_{A}\right)_{A \in \mathcal{C}},\left(f_{A}^{\prime}\right)_{A \in \mathcal{C}}$ are polynomial families; see $\S 1.1$. (Note that the definition of $f_{A}^{\prime}$ involves the isomorphisms $H, \tilde{H}$ in 2.8(a). By the proof of 2.8(a) these isomorphisms can be regarded as polynomial families when $A$ varies.) We can now apply Proposition 1.3 and we see that $f_{A}$ is bijective for any $A$. This proves 3.6(ii) for our $w$.

Now assume that $A, \chi, m, A^{\chi}$ are as in 3.6(iii). Let $A_{0}$ be an algebraic closure of $A_{1}:=A^{\chi}$. Let $A_{2}=A \otimes_{A_{1}} A_{0} \in \mathcal{C}$. Now $f_{A}: A^{N} \rightarrow A^{N}$ is not given by polynomials with coefficients in $A$; however, $A$ is an $A_{1}$-vector space of dimension $m$ and $f_{A}$ can be viewed as a map $A_{1}^{N m} \rightarrow A_{1}^{N m}$ given by polynomials with coefficients in $A_{1}$. The same polynomials describe the map $f_{A_{2}}: A_{2}^{N} \rightarrow A_{2}^{N}$ viewed as a map $A_{0}^{N m} \rightarrow A_{0}^{N m}$. This last map is injective by $\S 3.8$ (applied to $A_{2}$ ) and then it is automatically bijective by [BR] (see also [Ax], G1, 10.4.11]) applied to the affine space $A_{0}^{N m}$ over $A_{0}$. Thus $f_{A_{2}}$ is bijective. Let $\xi \in A^{N}$. Then $\xi^{\prime}:=f_{A_{2}}^{-1}(\xi) \in A_{2}^{N}$ is well defined. The Galois group of $A_{0}$ over $A_{1}$ acts on $A_{2}$ (via the action on the second factor) hence on $A_{2}^{N}$. This action is compatible with $f_{A_{2}}$ and it fixes $\xi$; hence it fixes $\xi^{\prime}$. Since $A_{1}$ is perfect it follows that $\xi^{\prime} \in\left(A \otimes_{A_{1}} A_{1}\right)^{N}=A^{N}$. We have $f_{A}\left(\xi^{\prime}\right)=\xi$. Thus $f_{A}$ is surjective, hence bijective. This proves 3.6(iii) for our $w$.

Now assume that $A, \chi$ are as in 3.6(iv). In this case $f_{A}$ can be viewed as a map $A^{N} \rightarrow A^{N}$ given by polynomials with coefficients in $A$. This map is injective by $\S 3.8$ and then it is automatically bijective by [BR] (see also [Ax, G1, 10.4.11]) applied to the affine space $A^{N}$. Thus $f_{A}$ is bijective. This proves 3.6(iv) for our $w$.

Finally assume that $A$ is finite. Then $A^{N}$ is finite. Since $f_{A}$ is injective, it is automatically bijective. This proves $3.6(\mathrm{v})$ for our $w$.

3.10. Now let $w$ be any element of $C_{\min }$.

Since $\Xi_{A}^{w^{\prime}}$ is injective for $w^{\prime}$ as in $\S 3.7$ (see $\S 3.8$ ) we see using $3.5(\mathrm{a})$ that $\Xi_{A}^{w}$ is injective. This proves 3.6(i) for our $w$.

Now assume that we are in the setup of 3.6(ii), (iii), (iv) or (v). Since $\Xi_{A}^{w^{\prime}}$ is bijective for $w^{\prime}$ as in $\S 3.7$ (see $\S 3.9$ ) we see using $3.5(\mathrm{a}),(\mathrm{b})$ that $\Xi_{A}^{w}$ is bijective. This completes the proof of Theorem 3.6.

3.11. Recall that $A \in \mathcal{C}$. In this subsection we assume that $\chi=1$. Let $w \in C_{\text {min }}$ ( $C$ as in 3.6). We identify $A^{N}=U_{A} \times\left(U_{A}^{w} \dot{w}\right)$ (with $N$ as in 3.6) as in 2.6(a), $2.7(\mathrm{e})$ and $A^{N}=U_{A} \dot{w} U_{A}$ as in $2.7(\mathrm{f})$. Then $\Xi_{A}^{w}$ becomes a map $A^{N} \rightarrow A^{N}$. From the definitions we see that $\left(\Xi_{A^{\prime}}^{w}\right)_{A^{\prime} \in \mathcal{C}}$ is a polynomial family. (Here $\delta$ is fixed and 
$\chi=1$ for any $A^{\prime}$.) Hence $\left(\Xi_{A}^{w}\right)^{*}: A\left[X_{1}, \ldots, X_{N}\right] \rightarrow A\left[X_{1}, \ldots, X_{N}\right]$ is defined for any $A \in \mathcal{C}$. We have the following result.

Theorem 3.12. In the setup of $\S 3.11,\left(\Xi_{A}^{w}\right)^{*}$ is an isomorphism. In particular, if $A$ is an algebraically closed field, then $\Xi_{A}^{w}: A^{N} \rightarrow A^{N}$ is an isomorphism of algebraic varieties.

Let $w^{\prime} \in C_{\text {min }}$ be as in $\S 3.7$. As we have seen in $\S 3.9$, there exists a polynomial family $\Xi_{A}^{\prime}: A^{N} \rightarrow A^{N}(A \in \mathcal{C})$ such that $\Xi_{A}^{\prime} \Xi_{A}^{w^{\prime}}=1$ for any $A$. Since $\Xi_{A}^{w^{\prime}}$ is bijective by 3.6(ii) we must also have $\Xi_{A}^{w^{\prime}} \Xi_{A}^{\prime}=1$. Now the method in the paragraph preceding 3.5(b) yields for any $A$ an explicit map $\Xi_{A}^{\prime \prime}: A^{N} \rightarrow A^{N}$ such that $\Xi_{A}^{w} \Xi_{A}^{\prime \prime}=1$. Moreover from the definitions we see that $\left(\Xi_{A}^{\prime \prime}\right)_{A \in \mathcal{C}}$ is a polynomial family. It follows that $\left(\Xi_{A}^{\prime \prime}\right)^{*}$ is defined and $\left(\Xi_{A}^{\prime \prime}\right)^{*}\left(\Xi_{A}^{w}\right)^{*}=1$. Since $\Xi_{A}^{w}$ is a bijection (see 3.6(ii)), we see that $\Xi_{A}^{w} \Xi_{A}^{\prime \prime}=1$ implies $\Xi_{A}^{\prime \prime} \Xi_{A}^{w}=1$; hence $\left(\Xi_{A}^{w}\right)^{*}\left(\Xi_{A}^{\prime \prime}\right)^{*}=1$. This, together with $\left(\Xi_{A}^{\prime \prime}\right)^{*}\left(\Xi_{A}^{w}\right)^{*}=1$, shows that $\left(\Xi_{A}^{w}\right)^{*}$ is an isomorphism. This completes the proof of the theorem. Note that the second assertion of the theorem can alternatively be proved using 1.2(ii) and the fact that $\Xi_{A}^{w}$ is injective (see 3.6(i)).

3.13. In this subsection we assume that $A, \chi$ are as in 3.6(iv). Let $w \in C_{\min }(C$ as in 3.6). We identify $A^{N}=U_{A} \times\left(U_{A}^{w} \dot{w}\right)$ (with $N$ as in 3.6) as in 2.6(a), 2.7(e) and $A^{N}=U_{A} \dot{w} U_{A}$ as in $2.7(\mathrm{f})$. Then $\Xi_{A}^{w}$ becomes a map $A^{N} \rightarrow A^{N}$. It is in fact a morphism of algebraic varieties. Exactly as in 3.12 we define a map $\Xi_{A}^{\prime \prime}: A^{N} \rightarrow A^{N}$ such that $\Xi_{A}^{w} \Xi_{A}^{\prime \prime}=1$. But this time $\Xi_{A}^{\prime \prime}$ is not a morphism of algebraic varieties but only a quasi-morphism (see [L5, 2.1]). (This is because the definition of $\Xi_{A}^{\prime \prime}$ involves $\chi^{-1}: A \rightarrow A$, which is a quasi-morphism but not a morphism.) Since $\Xi_{A}^{w}$ is a bijection (see 3.6(iv)) we deduce that we also have $\Xi_{A}^{\prime \prime} \Xi_{A}^{w}=1$. Thus we have the following result:

(a) $\Xi_{A}^{w}: A^{N} \rightarrow A^{N}$ is a bijective morphism whose inverse is a quasi-morphism.

3.14. Let $C$ be as in 3.6 and let $w \in C_{\min }$. Let $A \in \mathcal{C}$ and let $\delta, \chi, \pi$ be as in $\S 2.1$. We define

(a) $\alpha_{A}^{w}:{ }^{\delta-1}(w)^{-1} U_{A} \times U_{A}^{w} \rightarrow U_{A}$ by $\left(u^{\prime}, u^{\prime \prime}\right) \mapsto u^{\prime} u^{\prime \prime} \dot{w} \pi\left(u^{\prime}\right)^{-1} \dot{w}^{-1}$.

We show:

(b) $\alpha_{A}^{w}$ is injective.

Assume that

$$
\left(u^{\prime}, u^{\prime \prime}\right) \in \delta^{\delta^{-1}(w)^{-1}} U_{A} \times U_{A}^{w}, \quad\left(u_{1}^{\prime}, u_{1}^{\prime \prime}\right) \in \delta^{\delta^{-1}(w)^{-1}} U_{A} \times U_{A}^{w}
$$

and

$$
u^{\prime} u^{\prime \prime} \dot{w} \pi\left(u^{\prime}\right)^{-1} \dot{w}^{-1}=u_{1}^{\prime} u_{1}^{\prime \prime} \dot{w} \pi\left(u_{1}^{\prime}\right)^{-1} \dot{w}^{-1} .
$$

Then $\Xi_{A}^{w}\left(u^{\prime}, u^{\prime \prime} \dot{w}\right)=\Xi_{A}^{w}\left(u_{1}^{\prime}, u_{1}^{\prime \prime} \dot{w}\right)$. Using 3.6(i) we deduce $\left(u^{\prime}, u^{\prime \prime} \dot{w}\right)=\left(u_{1}^{\prime}, u_{1}^{\prime \prime} \dot{w}\right)$; hence $u^{\prime}=u_{1}^{\prime}, u^{\prime \prime}=u_{1}^{\prime \prime}$ and (a) follows.

We show:

(c) If $\chi, A$ are as in 3.6(ii),(iii),(iv) or (v), then $\alpha_{A}^{w}$ is bijective.

Let $u \in U_{A}$. By Theorem 3.6, we have $u \dot{w}=u^{\prime} u^{\prime \prime} \dot{w} \pi\left(u^{\prime}\right)^{-1}$ for some $u^{\prime} \in U_{A}, u^{\prime \prime} \in$ $U_{A}^{w}$. By 2.7(c) we can write $u^{\prime-1} u=u_{1} u_{2}$, where $u_{1} \in U_{A}^{w}, u_{2} \in{ }^{w} U_{A}$. Then 
$u_{1} \dot{w}\left(\dot{w}^{-1} u_{2} \dot{w}\right)=u^{\prime \prime} \dot{w} \pi\left(u^{\prime}\right)^{-1}$. Using $2.7(\mathrm{~d})$ we deduce that $\pi\left(u^{\prime}\right)^{-1}=\dot{w}^{-1} u_{2} \dot{w} \in$ ${ }^{w^{-1}} U_{A}$. Thus $u^{\prime} \in \delta^{-1}(w)^{-1} U_{A}$ so that $\alpha_{A}^{w}$ is surjective. Together with (b) this implies that $\alpha_{A}^{w}$ is bijective.

We note:

(d) If $\chi=1$ and $A$ is an algebraically closed field, then $\alpha_{A}^{w}$ is an isomorphism of algebraic varieties.

We note that $\left(\alpha_{A^{\prime}}^{w}\right)_{A^{\prime} \in \mathcal{C}}$ can be viewed as a polynomial family of injective maps $A^{\prime n} \rightarrow A^{\prime n}\left(A^{\prime} \in \mathcal{C}, n\right.$ as in $\left.\S 2.1\right)$. Hence the result follows from 1.2(ii).

\section{Applications}

4.1. In this section we assume that $A$ is an algebraically closed field. We write $G, U,{ }^{w} U, U^{w}, T$ instead of $G_{A}, U_{A},{ }^{w} U_{A}, U_{A}^{w}, T_{A}$. By [L2, 4.11], $G$ is naturally a connected reductive algebraic group over $A$ with root datum $\mathcal{R}$ and $U$ is the unipotent radical of a Borel subgroup $B^{*}$ of $G$ with maximal torus $T$ normalized by each $\dot{s}_{i}$. We assume that $G$ is semisimple or equivalenty that $\left\{i^{\prime} ; i \in I\right\}$ span a subgroup of finite index in $X$. Let $\delta$ be an automorphism of $\mathcal{R}$ (necessarily of finite order, say c). The corresponding group automorphism $\delta: G \rightarrow G$ (see $\S 2.10$ ) preserves the algebraic group structure and has finite order $c$. Let $\hat{G}$ be the semidirect product of $G$ with the cyclic group of order $c$ with generator $d$ such that $d x d^{-1}=\delta(x)$ for all $x \in G$. Then $\hat{G}$ is an algebraic group with identity component $G$. Let $\mathcal{B}$ be the variety of Borel subgroups of $G$. For each $w \in W$ let $\mathcal{O}_{w}$ be the set of all $\left(B, B^{\prime}\right) \in \mathcal{B} \times \mathcal{B}$ such that $B=x B^{*} x^{-1}, B^{\prime}=x \dot{w} B^{*} \dot{w}^{-1} x^{-1}$ for some $x \in G$. We have $\mathcal{B} \times \mathcal{B}=\bigsqcup_{w \in W} \mathcal{O}_{w}$. As in [L5, 0.1, 0.2], for any $w \in W$ let

$\mathfrak{B}_{w}=\left\{(g, B) \in G d \times \mathcal{B}:\left(B, g B g^{-1}\right) \in \mathcal{O}_{w}\right\}$,

$\tilde{\mathfrak{B}}_{w}=\left\{\left(g, g^{\prime w} U\right) \in G d \times G /{ }^{w} U: g^{\prime-1} g g^{\prime} \in \dot{w} U d\right\}$.

Define $\pi_{w}: \tilde{\mathfrak{B}}_{w} \rightarrow \mathfrak{B}_{w}$ by $\left(g, g^{\prime w} U\right) \mapsto\left(g, g^{\prime} B^{*} g^{\prime-1}\right)$.

In the remainder of this section we assume that $C$ is a $\delta$-elliptic $\delta$-conjugacy class in $W$ and that $w \in C_{m i n}$. Then $\pi_{w}$ is a principal bundle with group $T_{w}=\left\{t_{1} \in T\right.$ : $\left.\dot{w}^{-1} t \dot{w}=d t d^{-1}\right\}$, a finite abelian group (see loc.cit.); the group $T_{w}$ acts on $\tilde{\mathfrak{B}}_{w}$ by $t:\left(g, g^{\prime w} U\right) \mapsto\left(g, g^{\prime} t^{-1 w} U\right)$. Now $G$ acts on $\mathfrak{B}_{w}$ by $x:(g, B) \mapsto\left(x g x^{-1}, x B x^{-1}\right)$ and on $\tilde{\mathfrak{B}}_{w}$ by $x:\left(g, g^{\prime w} U\right) \mapsto\left(x g x^{-1}, x g^{\prime w} U\right)$. We show:

(a) Let $\mathcal{O}$ be a G-orbit in $\tilde{\mathfrak{B}}_{w}$. There is a unique $v \in U^{\delta(w)}$ such that $\left(\dot{w} v d,{ }^{w} U\right) \in \mathcal{O}$.

Clearly $\mathcal{O}$ contains an element of the form $\left(\dot{w} u d,{ }^{w} U\right)$, where $u \in U$. We first show the existence of $v$. It is enough to show that for some $z \in{ }^{w} U, v \in U^{\delta(w)}$ we have $z \dot{w} u d z^{-1}=\dot{w} v d$, that is, $u=\dot{w}^{-1} z^{-1} \dot{w} v \delta(z)$. Setting $z^{\prime}=\dot{w}^{-1} z^{-1} \dot{w}$, $w^{\prime}=\delta(w)$ we see that it is enough to show that $u=z^{\prime} v \dot{w}^{\prime} \delta\left(z^{\prime}\right)^{-1} \dot{w}^{\prime-1}$ for some $z^{\prime} \in \delta^{-1}\left(w^{\prime}\right)^{-1} U, v \in U^{w^{\prime}}$. But this follows from 3.14(c) with $\chi=1$ and $w$ replaced by $w^{\prime}$.

Now assume that $\left(\dot{w} v d,{ }^{w} U\right) \in \mathcal{O},\left(\dot{w} v^{\prime} d,{ }^{w} U\right) \in \mathcal{O}$, where $v, v^{\prime} \in U^{\delta(w)}$. We have $\dot{w} v^{\prime} d=u \dot{w} v d u^{-1}$ for some $u \in{ }^{w} U$. Setting $u^{\prime}=\dot{w}^{-1} u \dot{w} \in w^{-1} U$ we have $v^{\prime} d \dot{w}=u^{\prime} v d \dot{w} u^{\prime-1}$, that is, $v^{\prime}=u^{\prime} v \delta(\dot{w}) \delta\left(u^{\prime-1}\right) \delta(\dot{w})^{-1}$. Using 3.14(b) (appplied to $\delta(w)$ instead of $w$ ) we see that $u^{\prime}=1$ and $v=v^{\prime}$. This completes the proof of (a).

We can reformulate (a) as follows.

(b) The closed subvariety $\left\{\left(\dot{w} v d,{ }^{w} U\right): v \in U^{\delta(w)}\right\}$ of $\tilde{\mathfrak{B}}_{w}$ meets each $G$-orbit in $\tilde{\mathfrak{B}}_{w}$ in exactly one point. Hence the space of $G$-orbits in $\tilde{\mathfrak{B}}_{w}$ can be identified with the affine space $U^{\delta(w)}$. 
We show:

(c) The closed subvariety $\left\{\left(\dot{w} v d, B^{*}\right): v \in U^{\delta(w)}\right\}$ of $\mathfrak{B}_{w}$ is isomorphic to $U^{\delta(w)}$; its intersection with any $G$-orbit in $\mathfrak{B}_{w}$ is a single $T_{w}$-orbit (for the restriction of the $G$-action), hence is a finite nonempty set.

The first assertion of (c) is obvious. Now let $\overline{\mathcal{O}}$ be a $G$-orbit in $\mathfrak{B}_{w}$. Let

$$
Z=\left\{\left(\dot{w} v d, B^{*}\right) ; v \in U^{\delta(w)}\right\} \cap \overline{\mathcal{O}} .
$$

There exists a $G$-orbit $\mathcal{O}$ in $\tilde{\mathfrak{B}}_{w}$ such that $\pi_{w}(\mathcal{O})=\overline{\mathcal{O}}$. By (b) we can find $v \in$ $U^{\delta(w)}$ such that $\left(\dot{w} v d,{ }^{w} U\right) \in \mathcal{O}$. Then $\left(\dot{w} v d, B^{*}\right)=\pi_{w}\left(\dot{w} v d,{ }^{w} U\right) \in \overline{\mathcal{O}}$ so that $Z \neq \emptyset$. If $\left(\dot{w} v d, B^{*}\right) \in Z$ and $t \in T_{w}$, then $\left(t \dot{w} v d t^{-1}, B^{*}\right) \in \overline{\mathcal{O}}$ and $\left(t \dot{w} v d t^{-1}, B^{*}\right)=$ $\left(\dot{w} v^{\prime \prime} d,{ }^{w} U\right)$, where

$$
v^{\prime \prime}=\dot{w}^{-1} t \dot{w} v^{\prime} d t^{-1} d^{-1}=\left(d t d^{-1}\right) v^{\prime}\left(d t^{-1} d^{-1}\right) \in{ }^{w} U .
$$

Thus $\left(t \dot{w} v d t^{-1}, B^{*}\right) \in Z$ so that $T_{w}$ acts on $Z$.

Assume now that $v, v^{\prime} \in U^{\delta(w)}$ are such that $\left(\dot{w} v d, B^{*}\right) \in \overline{\mathcal{O}},\left(\dot{w} v^{\prime} d, B^{*}\right) \in \overline{\mathcal{O}}$. Then for some $x \in G$ we have

$$
\pi_{w}\left(x \dot{w} v d x^{-1}, x^{w} U\right)=\pi_{w}\left(\dot{w} v^{\prime} d,{ }^{w} U\right) .
$$

Since $\pi_{w}$ is a principal fibration with group $T_{w}$ it follows that

$$
\left(x \dot{w} v d x^{-1}, x^{w} U\right)=\left(\dot{w} v^{\prime} d, t^{-1 w} U\right)
$$

for some $t \in T_{w}$. Thus $\left(\dot{w} v d,{ }^{w} U\right),\left(t \dot{w} v^{\prime} d t^{-1},{ }^{w} U\right)$ are in the same $G$-orbit on $\tilde{\mathfrak{B}}_{w}$. Note that $\left(t \dot{w} v^{\prime} d t^{-1},{ }^{w} U\right)=\left(\dot{w} v^{\prime \prime} d,{ }^{w} U\right)$, where

$$
v^{\prime \prime}=\dot{w}^{-1} t \dot{w} v^{\prime} d t^{-1} d^{-1}=\left(d t d^{-1}\right) v^{\prime}\left(d t^{-1} d^{-1}\right) \in{ }^{w} U .
$$

Using (b) we deduce that $v=v^{\prime \prime}$. Thus

$$
\left(\dot{w} v^{\prime} d, B^{*}\right)=\left(\dot{w}\left(\dot{w}^{-1} t^{-1} \dot{w}\right) v\left(d t d^{-1}\right) d, B^{*}\right)=\left(t^{-1} \dot{w} v d t, B^{*}\right)=t^{-1}\left(\dot{w} v d, B^{*}\right)
$$

so that $\left(\dot{w} v^{\prime} d, B^{*}\right),\left(\dot{w} v d, B^{*}\right)$ are in the same $T_{w}$-orbit. This completes the proof of $(\mathrm{c})$.

We can reformulate (c) as follows.

(d) The closed subvariety $\left\{\left(\dot{w} v d, B^{*}\right): v \in U^{\delta(w)}\right\}$ of $\mathfrak{B}_{w}$ meets each $G$-orbit in $\mathfrak{B}_{w}$ in exactly one $T_{w}$-orbit. Hence the space of $G$-orbits in $\mathfrak{B}_{w}$ can be identified with the orbit space of the affine space $U^{\delta(w)}$ under an action of the finite group $T_{w}$.

Statements like the last sentence in (b) and (d) were proved in [L5, 0.4(a)] assuming that $G$ is almost simple of type $A, B, C$ or $D$. The extension to exceptional types is new.

4.2. In this subsection we assume that $\delta=1$ so that $d=1$. Let $\gamma$ be the unipotent class of $G$ attached to $C$ in $\mathrm{L} 3$. Recall from loc.cit. that $\gamma$ has codimension $l(w)$ in $G$. The following result exhibits a closed subvariety of $G$ isomorphic to the affine space $A^{l(w)}$ which intersects $\gamma$ in a finite set.

(a) The closed subvariety $\Sigma:=\dot{w} U^{w}$ of $G$ is isomorphic to $U^{w}$ and $\Sigma \cap \gamma$ is a single $T_{w}$-orbit (for the conjugation action), hence is a finite nonempty set.

According to [L4, the subset $\mathfrak{B}_{w}^{\gamma}=\left\{(g, B) \in \mathfrak{B}_{w}: g \in \gamma\right\}$ is a single $G$-orbit on $\mathfrak{B}_{w}$. Let $Z=\left\{\left(\dot{w} v, B^{*}\right): v \in U^{w}\right\} \cap \mathfrak{B}_{w}^{\gamma}, Z^{\prime}=\left\{\dot{w} v: v \in U^{w}\right\} \cap \gamma$. The first projection defines a surjective map $Z \rightarrow Z^{\prime}$. Since $Z$ is a single $T_{w^{-}}$-orbit (see 3.1(c)), it follows that $Z^{\prime}$ is a single $T_{w}$-orbit. This proves (a). 
[Note added 10.4.2011. It is likely that the intersection $\Sigma \cap \gamma$ in (a) is not transversal in bad characteristic.]

4.3. In this subsection we assume that $A, F_{q}, \chi$ are as in 3.6(iv). Then $\pi=\delta \chi$ : $G \rightarrow G$ is the Frobenius map for an $F_{q}$-rational structure on $G$. As in [DL, we set

$$
\tilde{X}_{w}=\left\{g^{\prime w} U \in G /{ }^{w} U: g^{-1} \pi\left(g^{\prime}\right) \in \dot{w} U\right\} .
$$

Now the finite group $G^{\pi}:=\{g \in G: \pi(g)=g\}$ acts on $\tilde{X}_{w}$ by $x: g^{\prime w} U \mapsto$ $x g^{\prime w} U$. Let ${ }^{w} U \backslash \backslash U$ be the set of orbits of the ${ }^{w} U$-action on $U$ given by $u_{1}$ : $u \mapsto \dot{w}^{-1} u_{1} \dot{w} u \pi\left(u_{1}\right)^{-1}$. According to [DL, 1.12], we have a bijection $G^{\pi} \backslash \tilde{X}_{w} \stackrel{\sim}{\longrightarrow}$ ${ }^{w} U \backslash \backslash U, g^{\prime w} U \mapsto \dot{w}^{-1} g^{-1} \pi\left(g^{\prime}\right)$ with inverse induced by $u \mapsto g^{\prime w} U$, where $g^{\prime} \in G$, $g^{\prime-1} \pi\left(g^{\prime}\right)=\dot{w} u$. Under the substitution $\dot{w}^{-1} u_{1} \dot{w}=u^{\prime}$, the ${ }^{w} U$-action above on $U$ becomes the ${ }^{w^{-1}} U$-action on $U$ given by $u_{2}: u \mapsto u^{\prime} u \delta(\dot{w}) p\left(u^{\prime}\right)^{-1} \delta(\dot{w})^{-1}$. Using $3.14(\mathrm{c})$ for $\delta(w)$ instead of $w$ we see that the space of orbits of this action can be identified with $U^{\delta(w)}$. Thus we have the following result.

(a) The space of orbits of $G^{\pi}$ on $\tilde{X}_{w}$ is quasi-isomorphic to the affine space $U^{\delta(w)}$.

A statement such as (a) was proved in [L5] assuming that $G$ is almost simple of type $A, B, C$ or $D$ and $\delta=1$. The extension to general $G$ is new.

\section{REFERENCES}

[Ax] J. Ax, Injective endomorphisms of algebraic varieties and schemes, Pacific J. Math. 31 (1969), 1-7. MR0251036 (40:4267)

[BR] A. Bialynicki-Birula and M. Rosenlicht, Injective morphisms of real algebraic varieties, Proc. Amer. Math. Soc. 13 (1962), 200-203. MR0140516 (25:3936)

[DL] P. Deligne and G. Lusztig, Representations of reductive groups over finite fields, Ann. of Math. (2) 103 (1976), 103-161. MR0393266 (52:14076)

[GP] M. Geck and G. Pfeiffer, Characters of finite Coxeter groups and representations of Iwahori-Hecke algebras, LMS Monographs, vol. 21, Oxford Univ. Press, 2000. MR.1778802 (2002k:20017)

[GKP] M. Geck, S. Kim and G. Pfeiffer, Minimal length elements in twisted conjugacy classes of finite Coxeter groups, J. Algebra 229 (2000), 570-600. MR.1769289 (2001h:20049)

[GM] M. Geck and J. Michel, Good elements of finite Coxeter groups and representations of Iwahori-Hecke algebras, Proc. London Math. Soc. 74 (1997), 275-305. MR.1425324 (97i:20050)

[G1] A. Grothendieck, Éléments de géométrie algébrique IV, Étude locale des schémas et des morphismes de schémas (Troisième partie), Inst. Hautes Étude Sci. Publ. Math. 28 (1966). MR0217086 (36:1781)

[G2] A. Grothendieck, Éléments de géométrie algébrique IV, Étude locale des schémas et des morphismes de schémas (Quatrième partie), Inst. Hautes Étude Sci. Publ. Math. 32 (1967). MR0238860 (39:220)

[He] X. He, Minimal length elements in some double cosets of Coxeter groups, Adv. Math. 215 (2007), 469-503. MR22355597 (2009g:20088)

[L1] G. Lusztig, Introduction to quantum groups, Progr. in Math. 110, Birkhäuser Boston, 1993. MR1227098 (94m:17016)

[L2] G. Lusztig, Study of a $\mathbf{Z}$-form of the coordinate ring of a reductive group, Jour. Amer. Math. Soc. 22 (2009), 739-769. MR2505299 (2010d:20055)

[L3] G. Lusztig, From conjugacy classes in the Weyl group to unipotent classes, Represent. Theory 15 (2011), 494-530. MR2833465

[L4] G. Lusztig, Elliptic elements in a Weyl group: a homogeneity property, arxiv:1007.5040.

[L5] G. Lusztig, On certain varieties attached to a Weyl group element, Bull. Inst. Math. Acad. Sinica (N.S.) 6 (2011), 377-414. 
[Se] A. Sevostyanov, Algebraic group analogues of the Slodowy slices and deformations of Poisson W-algebras, doi:10.1093/imrn/rnq139, Int. Math. Res. Notices (2011), 1880-1925. MR 2806525

[St] R. Steinberg, Regular elements of semisimple algebraic groups, Inst. Hautes Étude Sci. Publ. Math. 25 (1965), 49-80. MR0180554 (31:4788)

Department of Mathematics, Hong Kong University of Science and Technology, HONG KONG

Department of Mathematics, Massachusetts Institute of Technology, Cambridge, MASSACHUSETTS 02139-4307 